\title{
A support vector machine and a random forest classifier indicates a I5-miRNA set related to osteosarcoma recurrence
}

This article was published in the following Dove Press journal:

OncoTargets and Therapy

\author{
Yunfei $\mathrm{He}^{1,2, *}$ \\ Jun $\mathrm{Ma}^{\mathrm{l}, *}$ \\ An Wang ${ }^{1,3, *}$ \\ Weiheng Wang' \\ Shengchang Luo' \\ Yaoming $\mathrm{Liu}^{2}$ \\ Xiaojian $\mathrm{Ye}^{1}$
}

'Department of Orthopaedics, Changzheng Hospital Affiliated with Second Military Medical University, Shanghai, ${ }^{2}$ Department of Orthopaedics, Lanzhou General Hospital of Lanzhou Military

Command Region, Lanzhou, ${ }^{3}$ Department of Orthopaedics, Shanghai Armed Police Force Hospital, Shanghai, People's Republic of China

*These authors contributed equally to this work

Correspondence: Yaoming Liu Department of Orthopaedics, Lanzhou General Hospital of Lanzhou Military

Command Region, 333 Nanbinhe

Road, Lanzhou 730050, People's

Republic of China

Tel +86931899621I

Email 1812820335@qq.com

Xiaojian Ye

Department of Orthopaedics, Changzheng Hospital Affiliated with Second Military Medical University, 415 Fengyang Road, Shanghai 200003, People's Republic of China

Tel +86 2l 6053 |43|

Email xiye2012@I26.com
Background: Osteosarcoma, which originates in the mesenchymal tissue, is the prevalent primary solid malignancy of the bone. It is of great importance to explore the mechanisms of metastasis and recurrence, which are two primary reasons accounting for the high death rate in osteosarcoma.

Data and methods: Three miRNA expression profiles related to osteosarcoma were downloaded from GEO DataSets. Differentially expressed miRNAs (DEmiRs) were screened using MetaDE.ES of the MetaDE package. A support vector machine (SVM) classifier was constructed using optimal miRNAs, and its prediction efficiency for recurrence was detected in independent datasets. Finally, a co-expression network was constructed based on the DEmiRs and their target genes.

Results: In total, 78 significantly DEmiRs were screened. The SVM classifier constructed by 15 miRNAs could accurately classify 58 samples in 65 samples (89.2\%) in the GSE39040 database, which was validated in another two databases, GSE39052 (84.62\%, 22/26) and GSE79181 (91.3\%, 21/23). Cox regression showed that four miRNAs, including hsa-miR-10b, hsa-miR-1227, hsa-miR-146b-3p, and hsa-miR-873, significantly correlated with tumor recurrence time. There were 137, 147, 145, and 77 target genes of the above four miRNAs, respectively, which were assigned to 17 gene ontology functionally annotated terms and 14 Kyoto Encyclopedia of Genes and Genomes pathways. Among them, the "Osteoclast differentiation" pathway contained a total of seven target genes and was analyzed further.

Conclusion: The 15-miRNAs-based SVM classifier provides a potential useful tool to predict the recurrence of osteosarcoma. Our results suggest the possible mechanisms of osteosarcoma metastasis and recurrence and provide fresh DEmiRs as potential biomarkers or therapeutic targets for osteosarcoma.

Keywords: osteosarcoma, microRNA, lncRNA, SVM classifier, co-expression network

\section{Introduction}

Osteosarcoma, which originates in the mesenchymal tissue, is the most frequent primary solid malignancy of the bone. ${ }^{1}$ The high death rate of osteosarcoma may be attributed to metastasis and recurrence, especially pulmonary metastasis, and over $90 \%$ of patients with osteosarcoma die from pulmonary metastases. ${ }^{1,2}$ However, molecular mechanisms of metastasis and recurrence are still not well known. Therefore, it has a very important significance to understand the molecular mechanisms of metastasis and recurrence, and this will be helpful to develop novel prognostic biomarkers and targeted therapeutic agents for osteosarcoma. 
MicroRNAs (miRNAs) are noncoding small RNAs involved in gene regulation, which repress translation and cleave mRNAs by base-pairing to the $3^{\prime}$-untranslated region of the target genes, and thus participate in the regulation of various critical biological processes, including differentiation, progression, apoptosis, and proliferation of tumor cells. ${ }^{3}$ Recent research demonstrated that miRNAs played important roles in the occurrence and development of osteosarcoma, and there were abnormal expressions of many miRNAs in osteosarcoma. ${ }^{4,5}$ For example, downregulated miRNA-143 in osteosarcoma could promote apoptosis and suppress tumorigenicity by targeting Bcl-2, ${ }^{6}$ and downregulated miRNA$199 \mathrm{a}-3 \mathrm{p}$ in osteosarcoma could regulate cell proliferation and migration. ${ }^{7}$ In contrast, overexpression of miR-125b could suppress proliferation and migration of osteosarcoma cells through downregulation of STAT $3,{ }^{8}$ and overexpression of miR-451 in the osteosarcoma cells could significantly inhibit cell proliferation, migration, and tumorigenesis as well as promote cell apoptosis. ${ }^{5}$ A study by Maire et al found that five upregulated miRNAs were related to genes with low expression, whereas five downregulated miRNAs were related to genes with high expression, indicating that miRNAs provide a novel post-transcriptional mechanism for fine-tuning the expression of specific genes and pathways relevant to osteosarcoma. ${ }^{9}$ Although accumulating evidences have proved the important roles of various miRNAs in osteosarcoma, there remains a large amount of unknown miRNAs involved in osteosarcoma, and it is necessary to explore more novel miRNAs that can provide a new theoretical basis for development of targeted therapy for osteosarcoma.

In addition, a series of long non-coding RNAs (lncRNAs), which may be participants in the regulation of diverse cellular processes such as cell proliferation and apoptosis, stem cell pluripotency, and development, ${ }^{10-12}$ have been shown to be involved in osteosarcoma. For instance, MALAT1 promotes proliferation and metastasis of osteosarcoma cells by activating the PI3K/Akt pathway. ${ }^{13}$ Abnormal expressions of IncRNAs in osteosarcoma have been shown in many studies. For example, downregulation of lncRNA TUG1 inhibits osteosarcoma cell proliferation and promotes apoptosis, ${ }^{14}$ and decreased expression of IncRNA MEG3 could act as a potential predictor biomarker in the progression and poor prognosis of osteosarcoma. ${ }^{15}$ Overexpression of lncRNAs UCA1 and TUG1 correlates with poor prognosis in osteosarcoma, ${ }^{16,17}$ and upregulation of IncRNA HNF1A-AS1 promotes cell proliferation and metastasis in osteosarcoma through activation of the Wnt $\beta$-catenin signaling pathway. ${ }^{18}$ Increasingly, studies have evidenced that lncRNAs are capable of acting as either competitive endogenous RNAs for miRNAs or miRNA sponges, thus modulating miRNA activity. ${ }^{19,20}$ Moreover, it has been demonstrated that lncRNAs and miRNAs cooperate closely in fine-tuning gene expression transcriptionally and post-transcriptionally. ${ }^{21}$ Tan et al have provided evidence for miRNA-mediated interplay between lncRNAs and mRNAs in mouse embryonic stem cells. ${ }^{22}$ Recently, it has been reported that crosstalks between lncRNAs and miRNAs are crucial for cancer metastasis. ${ }^{23}$ However, interactions between IncRNAs and miRNAs have not been systematically investigated in osteosarcoma.

Furthermore, although more and more people devote effort to explore osteosarcoma-related miRNAs and lncRNAs, most of these studies focus on either miRNAs or lncRNAs, and underlying mechanisms remain unclear. Recent developments in bioinformatics and statistical genomics provide systems biology approaches, allowing for a better understanding of transcriptome organization and transcriptional regulation. Among these systems biology approaches, gene network analysis is a powerful approach that considers gene interactions and has been widely applied in gene expression studies of humans and model organisms. ${ }^{24-26}$

This study aimed to identify promising candidate miRNAs significantly associated with osteosarcoma recurrence and uncover the underlying molecular mechanisms. For doing this, we tried to use large quantities of miRNA-seq data in GEO DataSets to screen out miRNAs with differential expression between osteosarcoma recurrence and nonrecurrence samples, and then undertook correlational analysis between optimal feature miRNA combination and recurrence time. Finally, a co-expression network was constructed on the basis of the differential expression of miRNAs and their target genes. Moreover, lncRNAs related to osteosarcoma were searched in the lncRNA and disease (LncRNADisease) database, and the relationships between these lncRNAs and their target miRNAs were integrated using the two prediction databases - miRcode and starBase.

\section{Data and methods}

\section{Collection and pretreatment of expression profile data}

Public expression profiling data in GEO DataSets were retrieved with "osteosarcoma, homo sapiens and miRNA" as key words on July 25, 2016. Three miRNA expression profiles, named GSE39040, GSE39052, and GSE79181, were achieved, among which the former two emerged from the platform "GPL15762 Illumina Human v2 MicroRNA Expression BeadChip", whereas the last one arose from the 
platform "GPL15497 Applied Biosystems TaqMan Array Human MicroRNA Cards". There were 23, 15, and 14 osteosarcoma recurrence samples, and 42,11, and 9 osteosarcoma non-recurrence samples in these three datasets, separately. Meanwhile, original values of the abovementioned transcripts were downloaded in .txt format. Each probe was annotated as the corresponding gene after rejecting null probes, and then normalized by a limma package.

\section{Screening of differentially expressed miRNA (DEmiR) by meta-analysis}

DEmiRs between osteosarcoma recurrent and non-recurrent samples were screened using MetaDE.ES of the MetaDE package. ${ }^{27}$ First, heterogeneity tests of miRNA expression values from various platforms were carried out using MetaDE.ES to obtain statistical parameters for judging heterogeneity, including tau ${ }^{2}$, Q value, and Qpval. The judgment criteria were as follows: when the tau ${ }^{2}$ value was 0 or the $\mathrm{Q}$ value was submitted to the $\mathrm{K}-1$ degree of freedom chi-square test with Qpval $>0.05$, the study object was homogeneous and unbiased. Subsequently, a significant expression difference test $(P<0.05)$ of miRNA from the integrated dataset was conducted between different groups. In this study, sources of distinct miRNAs were ensured to be unbiased on the basis of reliable parameters of the homogeneity test (ie, $\operatorname{tau}^{2}=0$ and Qpval $>0.05$ ). Moreover, $P<0.05$ was chosen as a significant threshold of miRNA differential expression.

\section{Construction of specimen classification model using support vector machine (SVM) and random forest (RF)}

Acquisition of optimal feature miRNA combination

In the present study, GSE39040 was used as a training dataset, and hierarchical clustering analysis was undertaken based on the expression values of DEmiRs and the unsupervised clustering method. Moreover, the aforementioned miRNAs had undergone selection of optimal feature combinations using a recursive feature elimination (RFE) ${ }^{28}$ algorithm, applied in machine learning. Performances of different types of samples were evaluated through combinations of iterative random features until the optimal feature combination was obtained. Representative miRNAs were selected out after serial analyses, which can be used as identifiers of clinical diagnosis to construct a model and assess recurrent osteosarcoma based on expression levels of representative miRNAs.

\section{Construction of SVM classifier}

A significant combination of informative miRNAs was achieved using optimization of feature selection, and a SVM classification model was then constructed using the GSE39040 dataset by the e1071 package (https://cran.r-project.org/web/packages/ e1071/index.html). Samples were classified and discriminated according to expression values of their informative miRNAs; later, probabilities of samples belonging to some class were predicted and estimated on the basis of their eigen values, so as to distinguish and predict the risk of osteosarcoma recurrence based on these informative expression levels of miRNAs.

\section{Construction of RF classifier}

Furthermore, a RF classifier was established using the same informative miRNAs as in SVM classifier in the GSE39040 dataset by the randomForest package (https://cran.r-project. org/web/packages/randomForest/index.html). Samples were classified into different recurrence statuses and discriminated according to expression values of their informative miRNAs by the RF classifier.

\section{Validation of SVM and RF classifier}

In order to confirm the robustness and transferability of the SVM and RF classifier, the remaining two datasets GSE39052 and GSE79181 - were used as verification sets. The classification effect was comprehensively evaluated in terms of sensitivity (Se), specificity (Sp), positive predictive value (PPV), negative predictive value (NPV), and the area under the ROC curve (AUROC).

\section{Correlation analysis of selected miRNAs and recurrence time} Multiple analysis of optimal feature miRNAs and recurrence time

All samples in the training set were divided into a recurrence group and a non-recurrence group using the SVM classifier. Using Kaplan-Meier survival analysis of survfit function in $\mathrm{R}$ language, ${ }^{29}$ correlation analysis between optimal feature miRNAs and the recurrence time of each corresponding sample was undertaken in the two groups.

\section{Cox regression analysis of optimal feature miRNAs and recurrence time}

According to clinical information, all samples in the training set were categorized into a recurrence group and a nonrecurrence group. With expression values of the optimal feature combination of miRNAs as variables, associations of optimal feature miRNAs with recurrence time were studied 
by Cox regression analysis using the coxph $\mathrm{R}$ function of the survival package in $\mathrm{R}$ language.

\section{Prediction of miRNA-regulated target genes using miRTarBase database}

MiRTarBase (http://mirtarbase.mbc.nctu.edu.tw) provides the latest and extensive experimental validation of miRNA-target interaction information. ${ }^{29,30}$ Using the latest updated version of this database, the target genes of the miRNAs significantly associated with recurrence were searched. Subsequently, interaction networks of target genes regulated by these miRNAs were established after integration of the interaction data of human genes from the three protein-interaction databases, including the Biological General Repository for Interaction Datasets (BioGRID) (http://thebiogrid.org/), Human Protein Reference Database (HPRD) (http://www.hprd.org/), and the Database of Interacting Proteins (DIP) (http://dip.doe-mbi.ucla.edu/).

\section{Function and pathway of target genes in miRNA regulation network}

The miRNA control networks were visualized by Cytoscape. Later, the gene ontology (GO) function nodes and Kyoto Encyclopedia of Genes and Genomes (KEGG) pathways significantly enriched with the target genes were identified. The enrichment process was calculated by Fisher exact test algorithm, and the calculation formula was as follows:

$$
p=1-\sum_{i=0}^{x-1} \frac{\left(\begin{array}{c}
M \\
i
\end{array}\right)\left(\begin{array}{l}
N-M \\
K-i
\end{array}\right)}{\left(\begin{array}{l}
N \\
K
\end{array}\right)}
$$

$N$ is the total number of genes in the whole genome; $M$ is the number of genes in the pathway; and $K$ is the number of differentially expressed genes.

\section{Search of disease IncRNAs and regulation relationship}

Search for IncRNAs associated with osteosarcoma from database

LncRNAs related to osteosarcoma were searched in the LncRNADisease database ${ }^{31}$ (http://210.73.221.6/lncrnadisease) which contained 321 lncRNAs and 221 related diseases reported in more than 500 documents.

\section{Prediction of relationship between IncRNAs and target-regulating miRNAs}

The regulatory relation between lncRNAs and miRNAs was integrated using two prediction databases, miRecode (http:// www.mircode.org/) $)^{32}$ and starBase..$^{33}$ Then, the regulatory relationship between the osteosarcoma-related lncRNAs and the miRNA-targeted genes was built according to the previously obtained miRNAs relevant to this disease.

\section{Results \\ Screening of candidate miRNAs by meta-analysis}

The parametric values of each miRNA, including pval, tau $^{2}$, Qpval, and Qval, were calculated using the MetaDE package. According to the screening threshold described in the section "Data and methods", there were 78 significantly DEmiRs screened from the three datasets, of which the top ten miRNAs selected according to $P$-values were hsa-miR-432, hsa-miR-329, hsa-miR-889, hsa-miR-337-3p, hsa-miR-635, hsa-miR-296-3p, hsa-miR-548c-3p, hsamiR-625, hsa-miR-551a, and hsa-miR-510 (Table S1). Meanwhile, heat maps of the three datasets were drawn out by the heatmap.sig.genes function of the MetaDE package (Figure 1).

\section{Model construction of sample classification using SVM and RF}

Construction of SVM and RF classifier based on optimal feature miRNA combination

Hierarchical clustering analysis was carried out in the training dataset GSE39040 based on expression values of DEmiRs and an unsupervised clustering method. The results indicated that samples of the same type tended to gather together on the basis of the expression values of the 78 DEmiRs (Figure 2A). In order to obtain a collection of miRNAs representative of all the DEmiRs the RFE algorithm was adopted to select the optimal characteristic combination of miRNAs with a significant difference in expression in the GSE39040 dataset. Following serial analyses, the result showed that the optimal miRNA combination had the highest classification accuracy of $89.2 \%$ when the number of miRNA was set at 15 (Figure 2B). These 15 miRNAs in the optimal combination were hsa-miR-10b, hsa-miR-146b-3p, hsa-miR-1324, hsa-miR-873, hsa-miR-1275, hsa-miR-129-3p, hsa-miR-124, hsa-miR-200c, hsa-miR-134, hsa-miR-139-3p, hsa-miR-122, hsa-miR-1227, hsa-miR-136, hsa-miR-127-3p, and hsa-miR885-3p (Table 1).

Using these 15 miRNAs in the optimal combination, analysis of two-way hierarchical clustering was applied to all samples ( $\mathrm{n}=65$ ) in database GSE39040, and the result was similar to that in Figure 2A; namely, samples of the same type also tended to gather together (Figure 3A). Moreover, the SVM classifier constructed by these 15 miRNAs could 


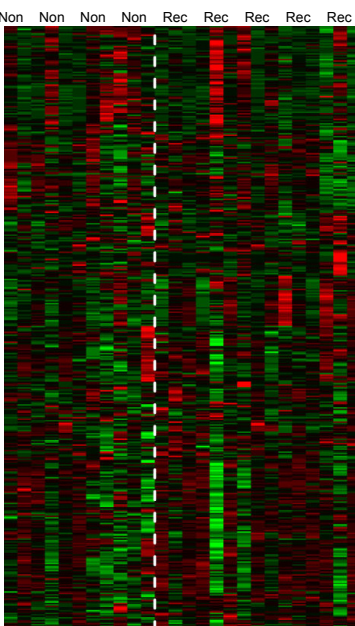

GSE39052

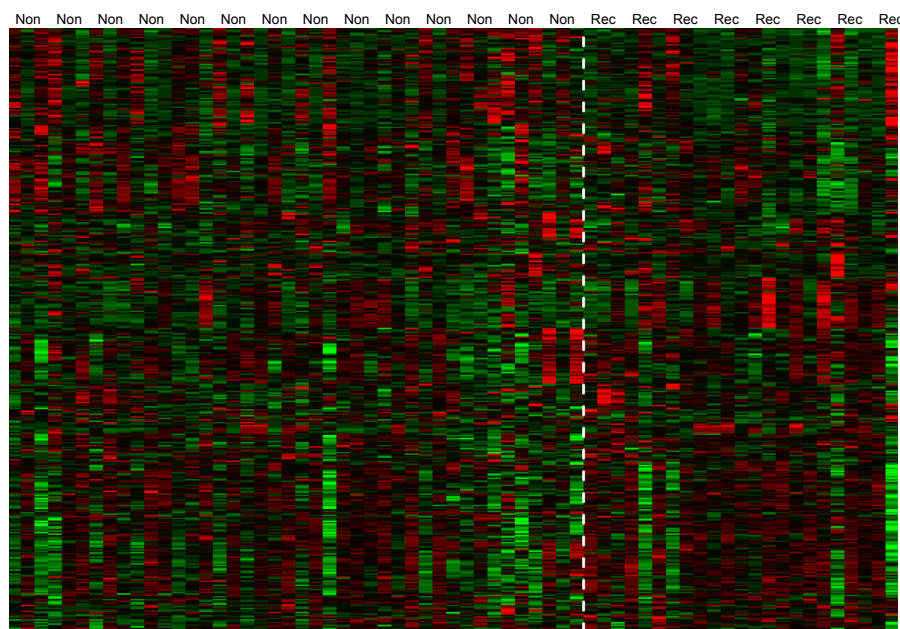

GSE39040

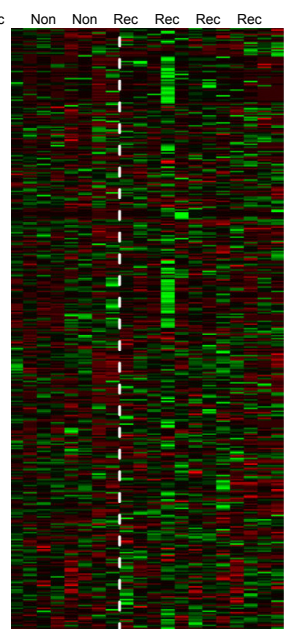

GSE79181

Figure I Heat maps of datasets GSE39052, GSE39040, and GSE79 I8I.

accurately classify 58 of the 65 samples. The classification scatter diagram and ROC chart of samples are shown in Figure $3 \mathrm{~B}$ and $\mathrm{C}$, respectively.

For the RF classifier, those 15 miRNAs could accurately classify 60 of the 65 samples, including 21 recurrence samples and 39 non-recurrence samples. The RF classification confusion table diagram and the ROC chart of samples are shown in Figure 4A and B, respectively.

\section{Independent validation and evaluation of SVM classifier performance}

GSE39052 and GSE79181, the remaining two datasets, were regarded as the verification sets to confirm robustness and transferability of the constructed classifier. The results showed that the SVM classifier categorized 22 samples precisely in GSE39052, with a classification accuracy rate of $84.62 \%(22 / 26)$; 21 of 23 samples were accurately classified in GSE79181, with $91.3 \%$ accuracy rate. The scatter diagrams and ROC charts of the two abovementioned datasets are presented in Figure 5.

Moreover, the RF classifier was validated in GSE39052 and GSE79181 datasets, which also showed high accuracy in recurrence status classification. The accuracy were $84.62 \%$ $(22 / 26)$ in GSE39052 and 91.3\% (21/23) in GSE79181 (Figure 6).

Furthermore, the sample classification effect of the SVM and RF classifier after training was evaluated using a variety of indicators, such as "Correct Rate", "Sensitivity", "Specificity", "PPV", "NPV", and "AUROC", which are described in Table 2.
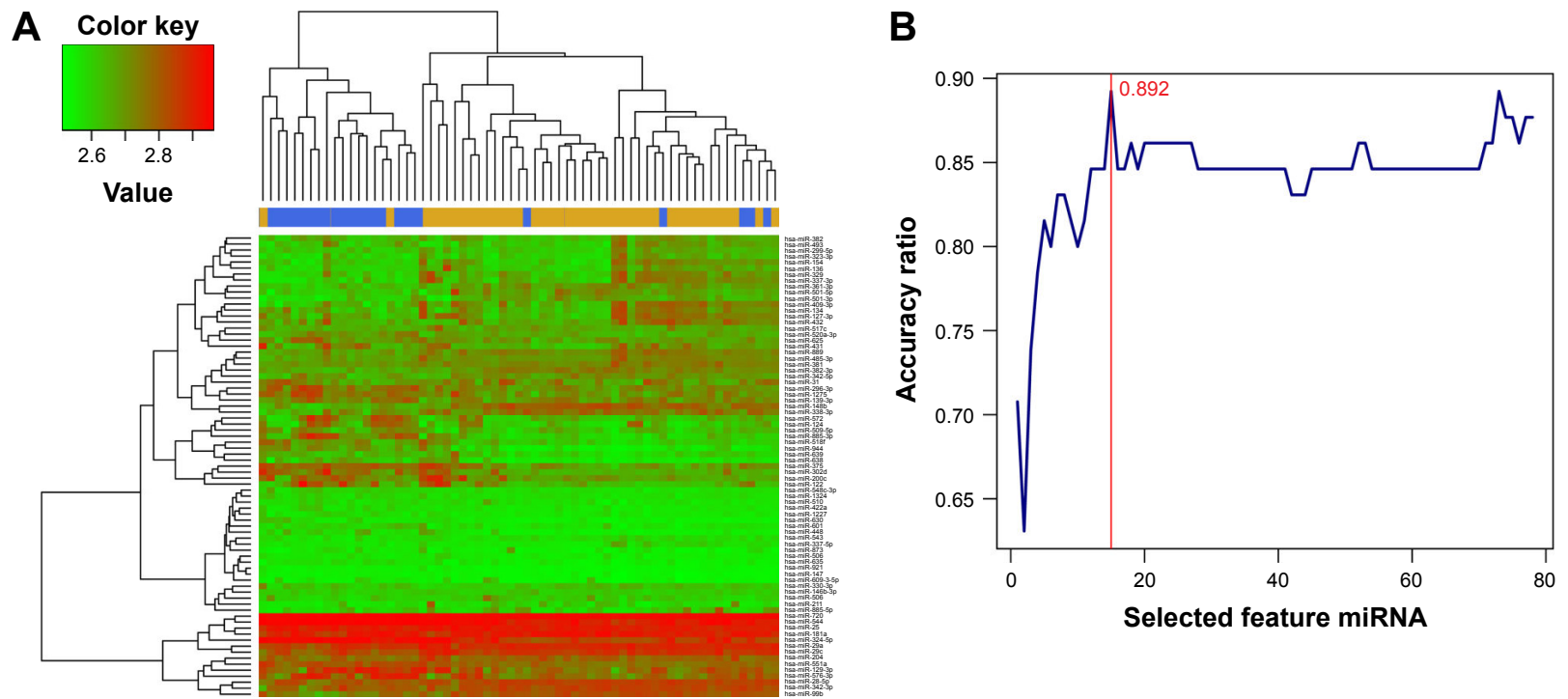

Figure 2 Bidirectional hierarchical clustering of 78 miRNAs based on their expression value (A) and feature elimination curves of 78 miRNA (B). 
Table I Statistical parameters of 15 miRNAs of optimal combination

\begin{tabular}{lllll}
\hline miRNA_ID & $P$-value & $\mathbf{Q}$ & Qp & tau $^{2}$ \\
\hline hsa-miR-I0b & 0.0009 & 1.6776 & 0.4322 & 0 \\
hsa-miR-I46b-3p & 0.0083 & 0.1552 & 0.9253 & 0 \\
hsa-miR-I324 & 0.0092 & 0.2516 & $0.88 I 8$ & 0 \\
hsa-miR-873 & 0.0237 & $0.158 I$ & 0.9240 & 0 \\
hsa-miR-I275 & 0.0296 & 0.2700 & 0.8737 & 0 \\
hsa-miR-I29-3p & 0.0326 & 0.6742 & 0.7138 & 0 \\
hsa-miR-I24 & 0.0338 & 0.7222 & 0.6969 & 0 \\
hsa-miR-200c & 0.0365 & 0.9670 & 0.6166 & 0 \\
hsa-miR-I34 & 0.0390 & 1.2245 & 0.5421 & 0 \\
hsa-miR-I39-3p & 0.0407 & 1.7569 & 0.4154 & 0 \\
hsa-miR-I22 & 0.0425 & 0.0150 & 0.9925 & 0 \\
hsa-miR-I227 & 0.0437 & 0.4731 & 0.7894 & 0 \\
hsa-miR-I36 & 0.0441 & 0.4049 & 0.8167 & 0 \\
hsa-miR-I27-3p & 0.0470 & 0.2269 & 0.8928 & 0 \\
hsa-miR-885-3p & 0.0494 & 0.8097 & 0.6671 & 0 \\
\hline
\end{tabular}

\section{Correlation analysis between optimal} feature miRNA combination and recurrence time

Expression values of the optimal feature miRNAs and recurrence time of each corresponding sample were obtained in the GSE39040 dataset, and all samples in the set were divided into a non-recurrence group and a recurrence group by the constructed SVM model. Kaplan-Meier survival analysis was then carried out for the two groups. The result showed that the survival time of the non-recurrence group was significantly longer compared to the recurrence group $(P=4.61 \mathrm{e}-05$; Figure 7$)$.

In addition, using expression values of the optimal 15 miRNAs as variables, their correlation with the recurrence
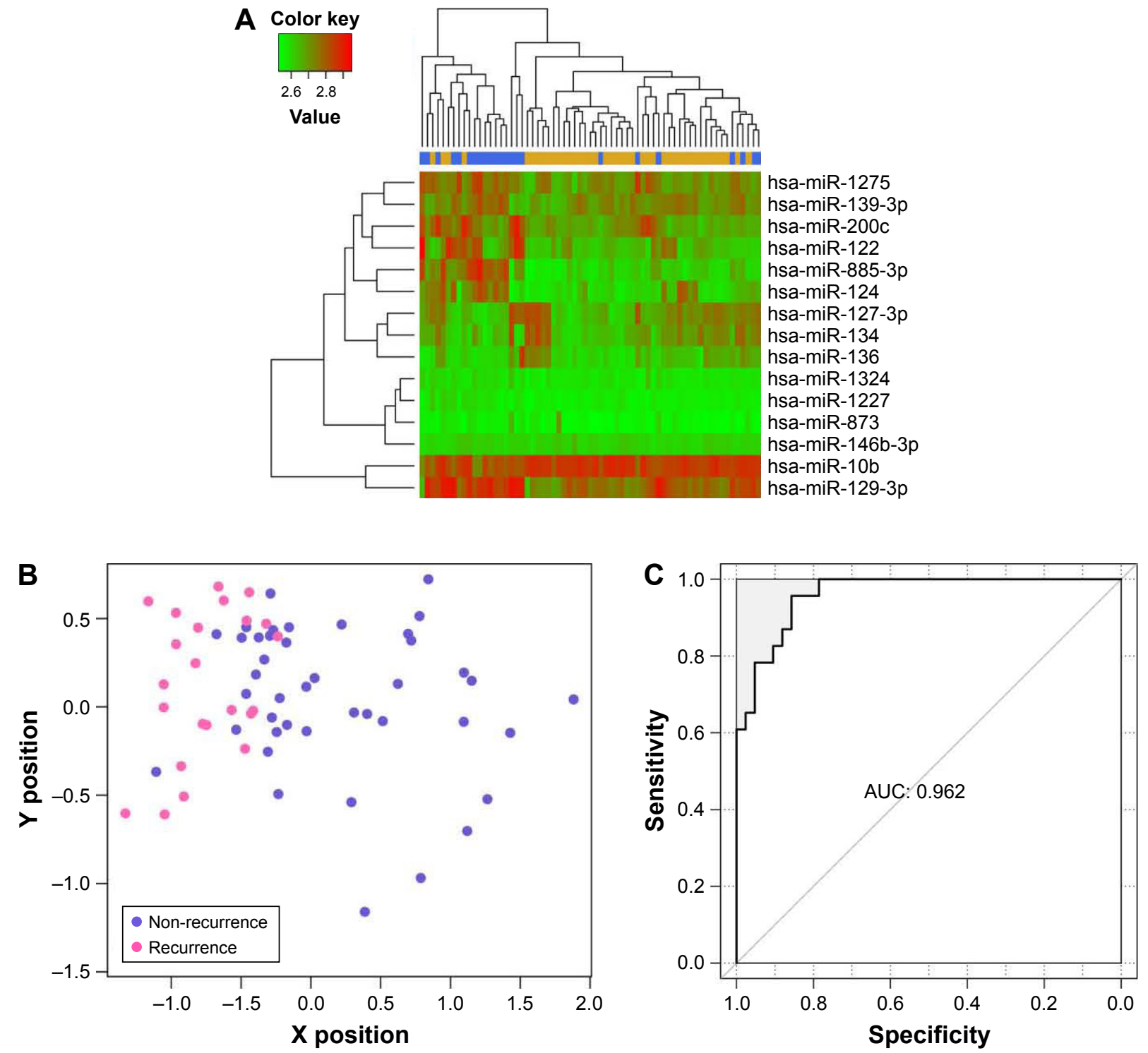

Figure 3 Bidirectional hierarchical clustering of 15 miRNAs from the optimal miRNA combination (A), scatter diagram (B), and ROC chart (C) of GSE39040 samples using the SVM classifier.

Abbreviations: AUC, area under curve; SVM, support vector machine. 
A

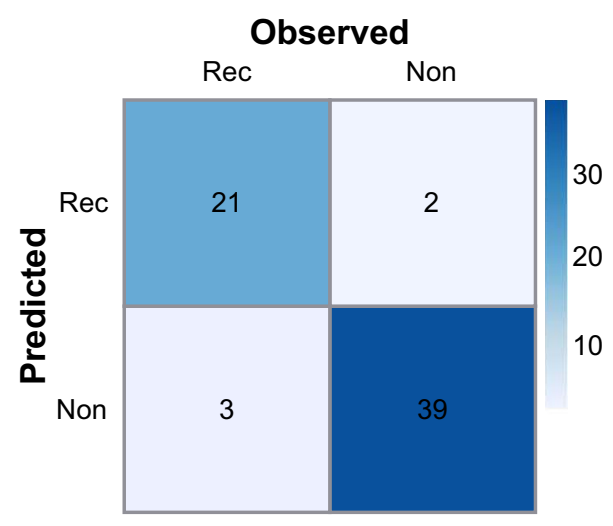

B

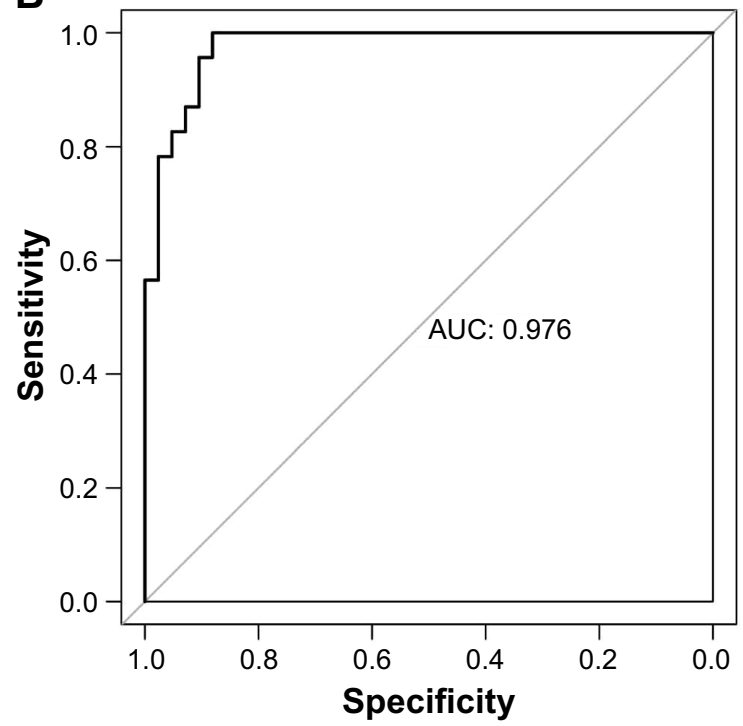

Figure 4 Confusion table diagram (A) and ROC chart (B) of GSE39040 samples using the RF classifier.

Abbreviations: AUC, area under curve; Non, non-recurrence; Rec, recurrence; RF, random forest; ROC, receiver operating characteristic curve.

A
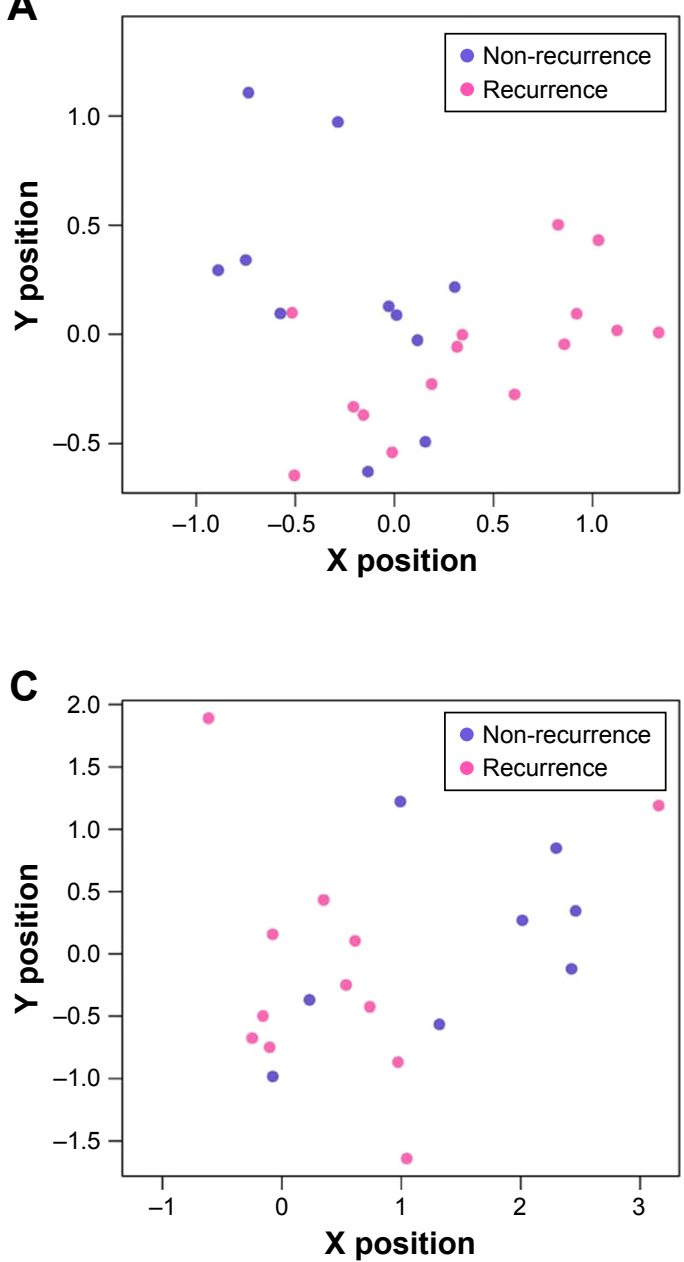

B

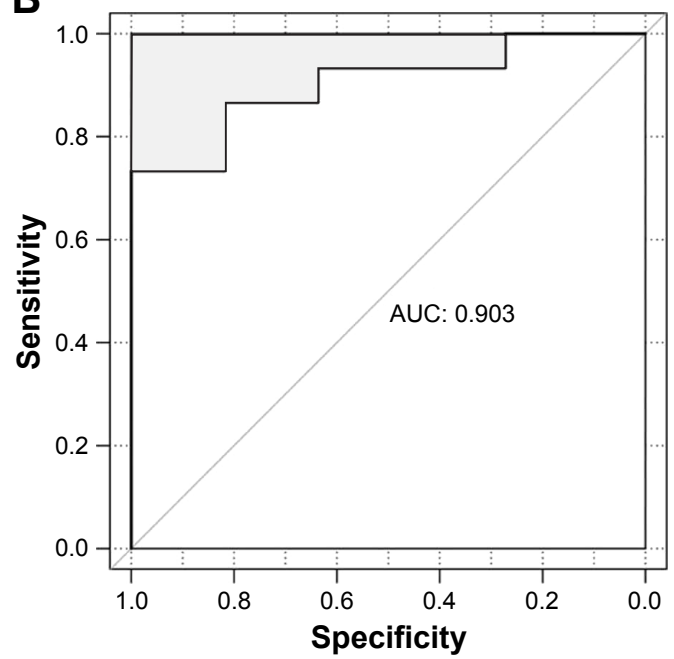

D

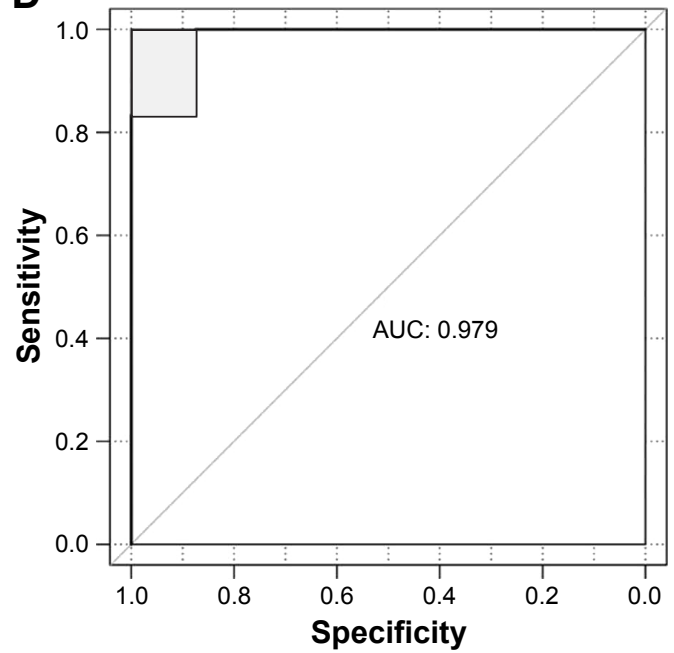

Figure 5 Scatter diagram and ROC chart of classification by the SVM classifier in validation datasets GSE39052 (A, B) and GSE79I8I (C, D). Abbreviations: AUC, area under curve; ROC, receiver operating characteristic curve; SVM, support vector machine. 
A

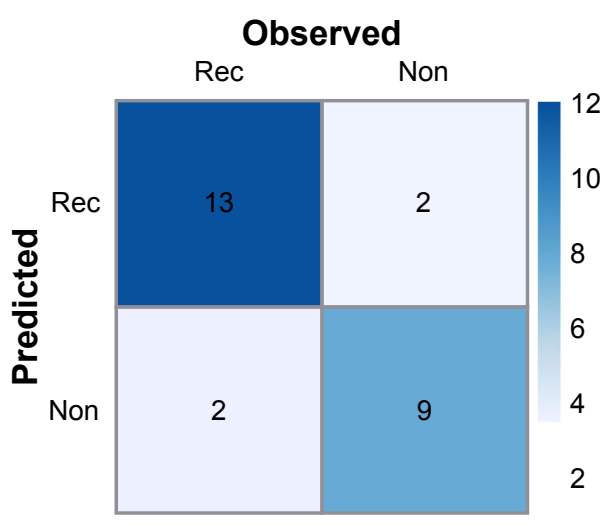

C

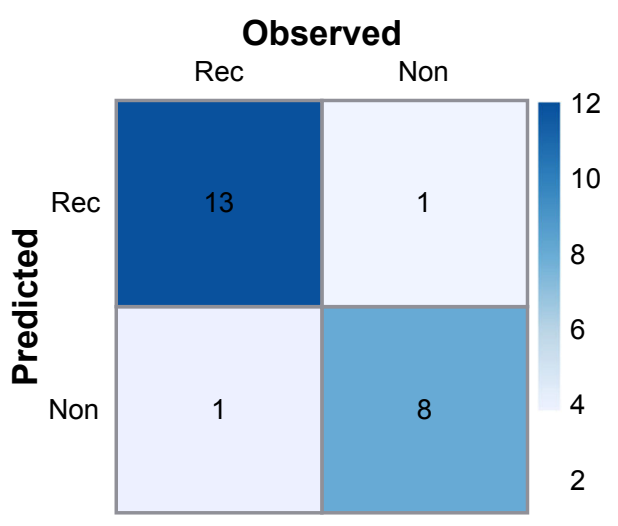

B

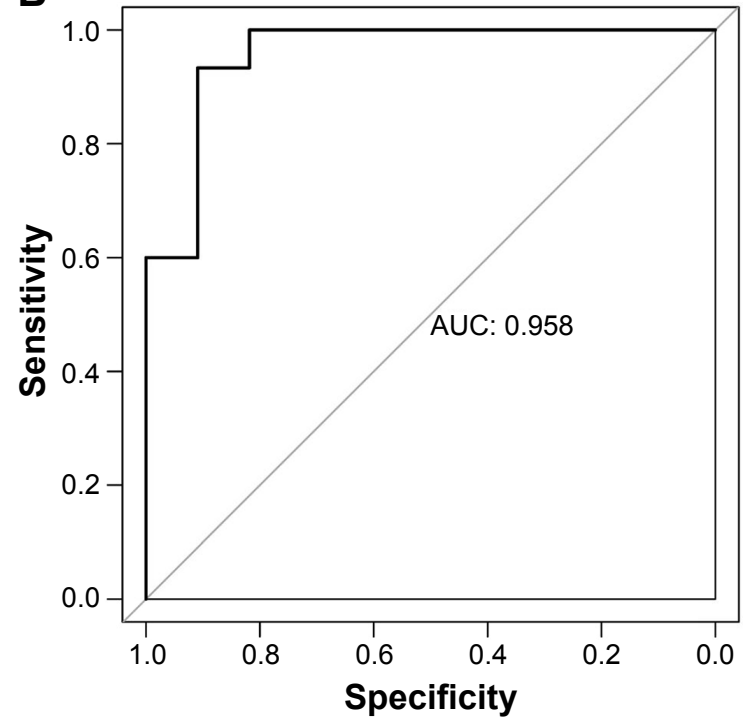

D

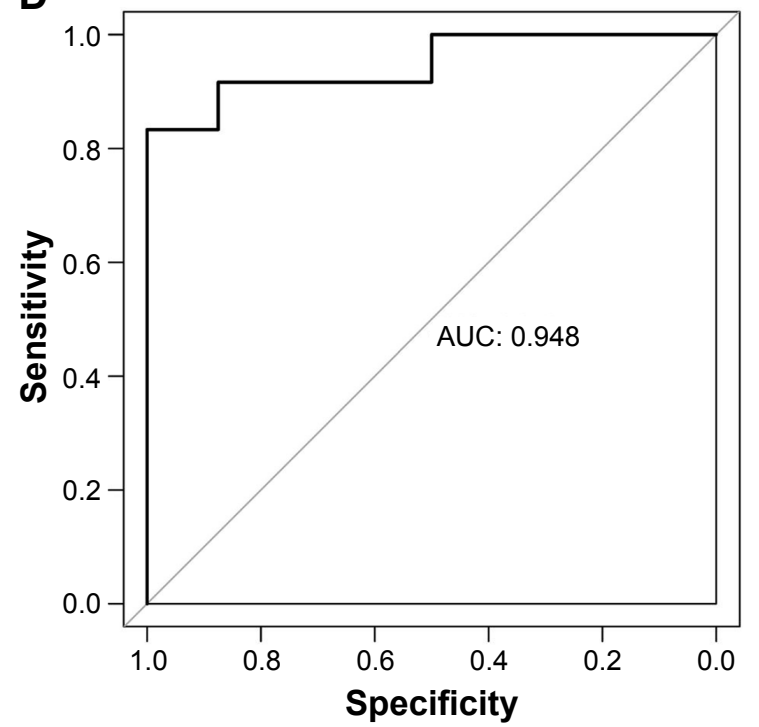

Figure 6 Confusion table diagram (A) and ROC chart (B) by the RF classifier in validation datasets GSE39052 (A, B) and GSE79I8I (C, D).

Abbreviations: AUC, area under curve; Non, non-recurrence; Rec, recurrence; RF, random forest; ROC, receiver operating characteristic curve.

time and sample status (recurrence or non-recurrence) hsa-miR-146b-3p, and hsa-miR-873 (Table 3). Kaplan-Meier according to clinical information was analyzed by Cox survival curve analysis was conducted to validate the signifiregression analysis. Four miRNAs were significantly corre- cant relevance between the expression of the four miRNAs lated with recurrence, including hsa-miR-10b, hsa-miR-1227, and recurrence (Figure 8). With median expression of each of

Table 2 List of index items used in evaluating classification effect of the SVM and RF classifier in the test datasets

\begin{tabular}{|c|c|c|c|c|c|c|c|}
\hline Datasets & $\begin{array}{l}\text { Number of } \\
\text { samples }\end{array}$ & Correct rate & Sensitivity & Specificity & PPV & NPV & AUROC \\
\hline \multicolumn{8}{|l|}{ SVM classifier } \\
\hline GSE39040 & 65 & 0.892 & 0.87 & 0.905 & 0.833 & 0.927 & 0.962 \\
\hline GSE39052 & 26 & 0.846 & 0.867 & 0.818 & 0.867 & 0.818 & 0.903 \\
\hline GSE79I8I & 23 & 0.913 & 0.929 & 0.889 & 0.929 & 0.889 & 0.979 \\
\hline \multicolumn{8}{|l|}{ RF classifier } \\
\hline GSE39040 & 65 & 0.923 & 0.875 & 0.951 & 0.913 & 0.929 & 0.976 \\
\hline GSE39052 & 26 & 0.846 & 0.867 & 0.818 & 0.867 & 0.818 & 0.958 \\
\hline GSE79|8I & 23 & 0.913 & 0.927 & 0.889 & 0.929 & 0.889 & 0.948 \\
\hline
\end{tabular}

Abbreviations: AUROC, area under the ROC curve; PPV, positive predictive value; NPV, negative predictive value; RF, random forest; SVM, support vector machine; ROC, receiver operating characteristic curve. 


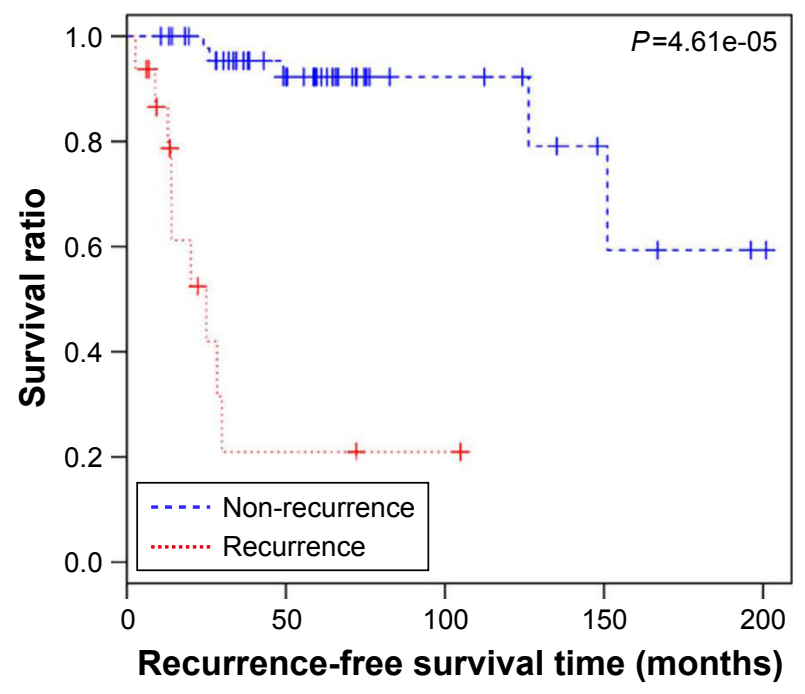

Figure 7 Kaplan-Meier survival curve between recurrence and non-recurrence samples.

the four miRNAs as the cutoff, all samples in the GSE39040 dataset were divided into two groups, separately. With regard to each miRNA, differences in the recurrence-free survival time between the patients with above-median value and the patients with below-median value were significant $(P<0.05)$.

\section{Interaction network analysis of target genes regulated by miRNA}

Target genes of the four abovementioned miRNAs, including hsa-miR-10b, hsa-miR-1227, hsa-miR-146b-3p, and hsamiR-873, were discovered from the miRTarBase database, whose gene numbers were $137,147,145$, and 77 , separately. The interactive relationships among these target genes were comprehensively searched in the three protein databases as described in the section "Data and methods", and 141 pairs of gene links were obtained. Interaction networks of target genes under regulation of the four miRNAs were built by integrating two kinds of connections - miRNA-gene and gene-gene. The network contained two types of nodes after visualization by Cytoscape: compared with the non-recurrence group, a red square node represented a miRNA with upregulated expression in the recurrence group, and a green square node stood for a miRNA with downregulated expression in the

Table 3 Results of Cox regression analysis

\begin{tabular}{llll}
\hline miRNA & Hazard ratio & $\mathbf{9 5 \%} \mathbf{~ C l}$ & P-value \\
\hline hsa-miR-I0b & 3.354 & $1.314-8.56$ & 0.0062 \\
hsa-miR-I227 & 1.337 & $0.583-3.066$ & 0.0423 \\
hsa-miR-I46b-3p & 2.203 & $0.932-5.209$ & 0.0093 I \\
hsa-miR-873 & 0.516 & $0.222-1.203$ & 0.0107 \\
\hline
\end{tabular}

recurrence group. Moreover, pink rounds indicated target genes (Figure 9).

\section{Function annotation and pathway enrichment of target genes in miRNA regulation network}

The functions and pathways of target genes involved in the above interaction networks were enriched by the hypergeometric distribution pathway enrichment analysis using Fisher algorithm. The results in Figure 10 indicated 14 significant KEGG pathways (Figure 10A) and 17 significant GO function terms (Figure 10B and Table S2). Among these significant KEGG pathways, one named the "Osteoclast differentiation" pathway was considered to be closely related to osteoblasts and osteosarcoma, and significantly involved a total of seven target genes, including Interleukin 1 alpha (IL1A), CAMK4, TNFSF11, NCF4, PPP3CB, MAP3K7, and suppressor of cytokine signaling (SOCS) 3 (Table S3). They were validated to be targeted by hsa-miR-10b, hsa-miR-1227, and hsa-miR-146b-3p, separately, in this study.

\section{Searching of osteosarcoma-related IncRNAs and establishment of relationships among IncRNA, miRNA, and target genes} Three lncRNAs - named BC040587, ${ }^{34}$ LSAMP-AS3, ${ }^{35}$ and MALAT $1^{13}$ - were checked for an association with osteosarcoma in the LncRNADisease database. miRNAs regulated by the three IncRNAs were searched in the miRecode and starBase databases and, afterward, compared with the four obtained important miRNAs in hsa-miR-10b, hsa-miR-1227, hsa-miR-146b-3p, hsa-miR-873. A regulatory network of the IncRNA-miRNA-target gene pathway was built by integrating various relationships between lncRNA and miRNAs, miRNAs and target genes, and target genes involved in pathways (Figure 11). In the regulatory network, MALAT1 may directly regulate hsa-miR-146b-3p, which imposed a regulatory effect on the $I L 1 A$ gene involved in the osteogenic differentiation pathway related to osteosarcoma.

\section{Discussion}

Metastasis and recurrence are the main factors that affect the prognosis of osteosarcoma, and involve a series of functional and regulating factors, such as miRNAs. Recent research demonstrated that miRNAs played important roles in the occurrence and development of osteosarcoma, and there were abnormal expressions of many miRNAs in osteosarcoma. ${ }^{4-9}$ There was great significance and relevance in comparing the expression of miRNAs between osteosarcoma recurrence 
A
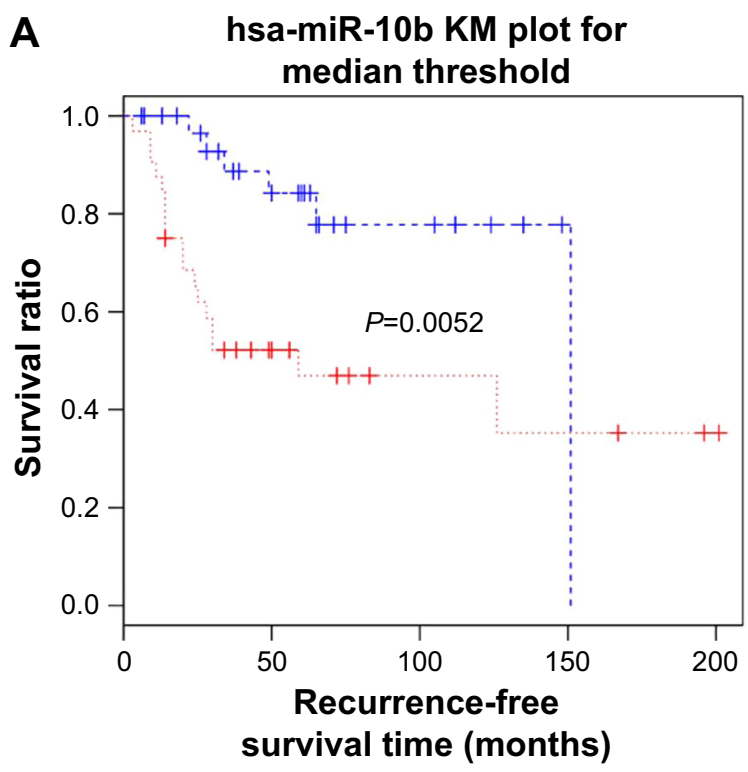

C

hsa-miR-873 KM plot for median threshold

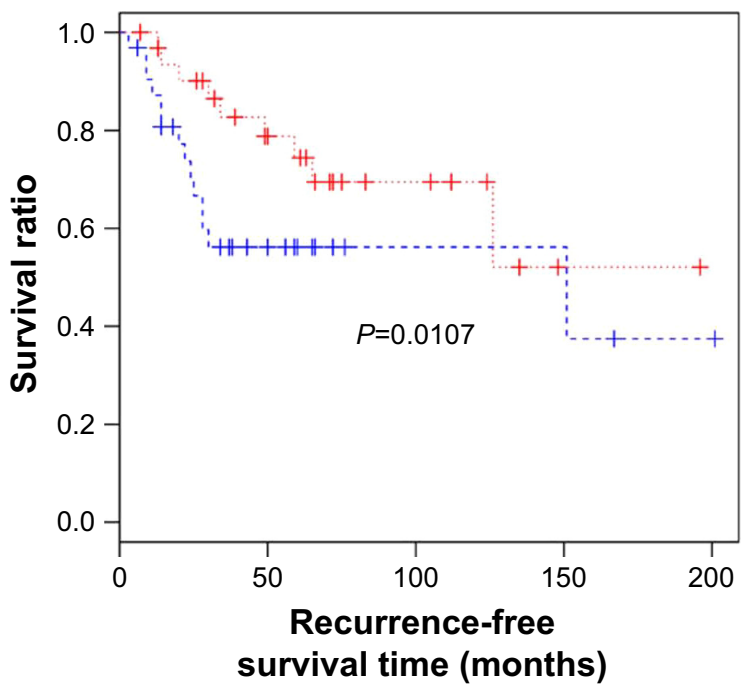

B hsa-miR-146b-3p KM plot for median threshold

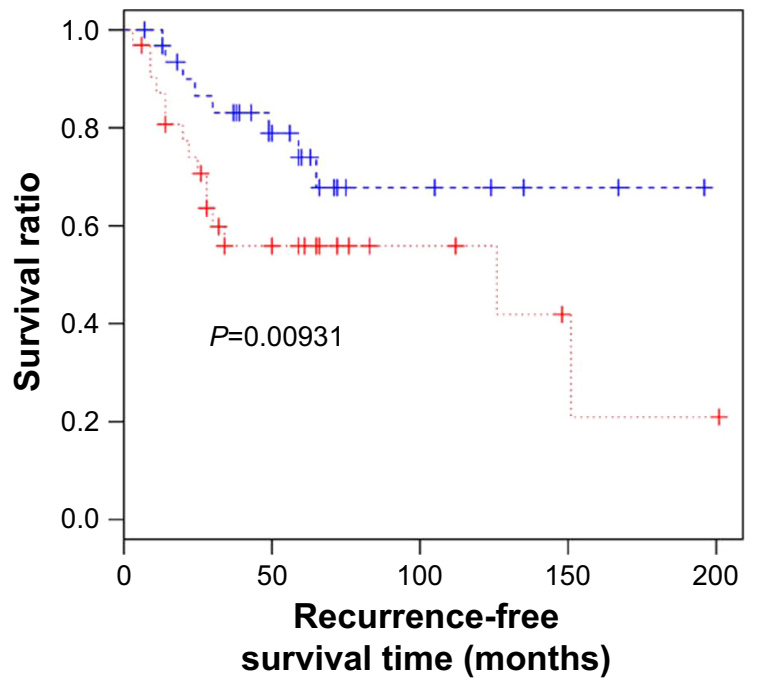

D hsa-miR-1227 KM plot for median threshold

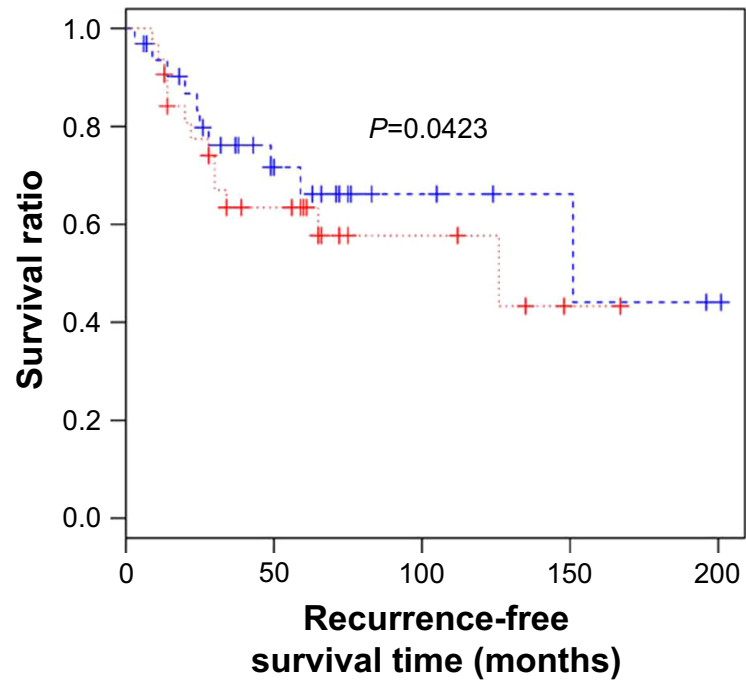

$\ldots$. Below median $(\mathrm{n}=62) \cdots \cdots$ Above median $(\mathrm{n}=62)$

Figure 8 Kaplan-Meier curves of hsa-miR-10b (A), hsa-miR-146b-3p (B), hsa-miR-873 (C), and hsa-miR-1227 (D), which are significantly associated with recurrence information.

samples and osteosarcoma non-recurrence samples. In the present study, three miRNA expression profiles related to osteosarcoma recurrence samples and osteosarcoma nonrecurrence samples - named GSE39052, GSE39040, and GSE79181 - were first downloaded from GEO DataSets, and then the DEmiRs were screened using MetaDE.ES of the MetaDE package. ${ }^{27}$ In total, 78 significantly DEmiRs were screened from the three datasets, and hierarchical clustering analysis on the basis of these miRNAs expression values indicated that the same-type samples in the GSE39040 dataset tended to gather together. The top ten DEmiRs were hsa-miR-432, hsa-miR-329, hsa-miR-889, hsa-miR-337-3p,
hsa-miR-635, hsa-miR-296-3p, hsa-miR-548c-3p, hsa-miR625, hsa-miR-551a, and hsa-miR-510. Among these, hsamiR-337-3p, ${ }^{36}$ miR- $625^{37}$, and miR-551 $\mathrm{a}^{38}$ were reported to be associated with lymph node metastasis of human gastric cancer, indicating the potential relationship between these miRNAs and osteosarcoma.

The SVM, a supervised machine-learning technique, has been shown to perform well in multiple areas of biological analysis, including evaluating microarray expression data, ${ }^{39}$ and in analyzing expression data. ${ }^{40,41}$ In the present study, in order to construct a specimen classification model using the SVM, the selection of optimal feature miRNA 


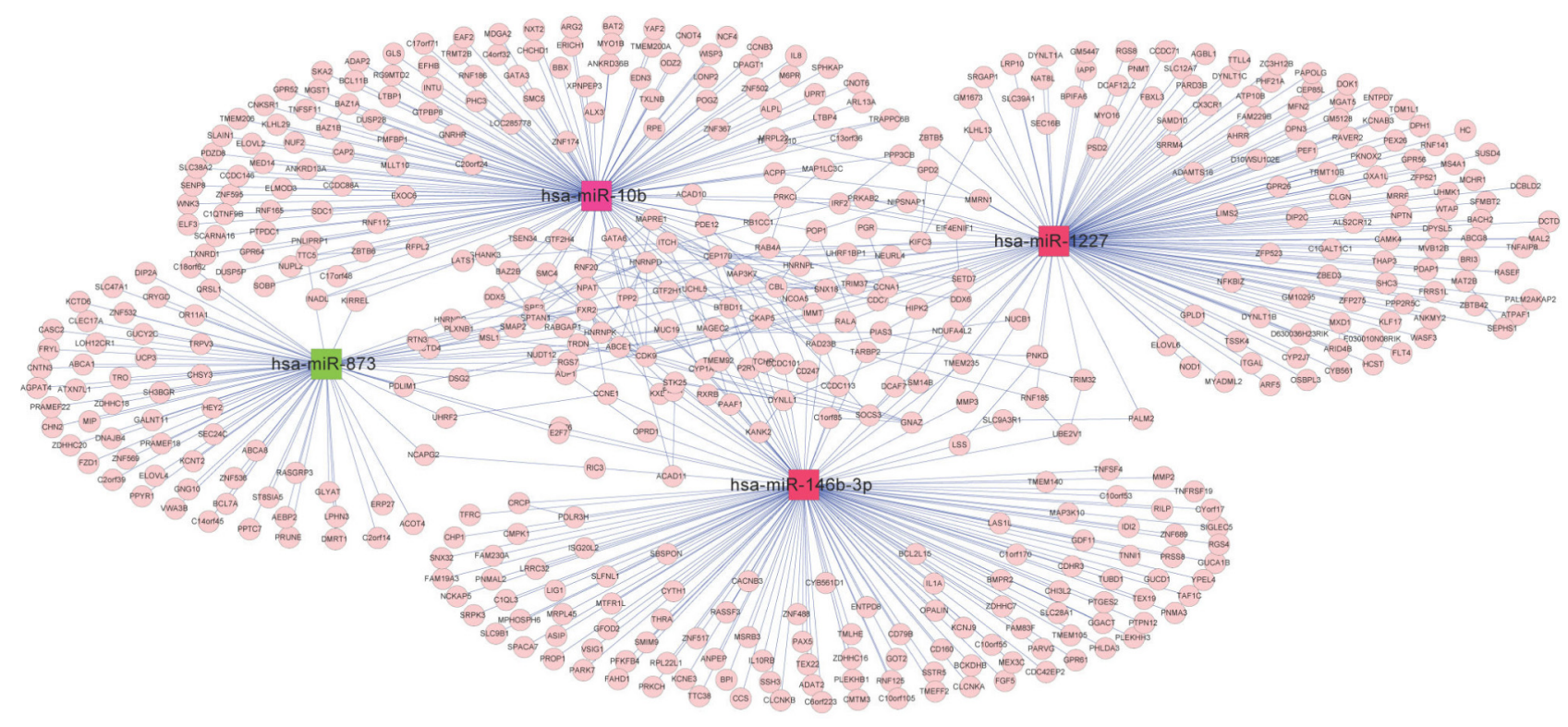

Figure 9 Interaction network among target genes of hsa-miR-1 0b, hsa-miR-1227, hsa-miR-146b-3p, and hsa-miR-873. A pink circle indicates a target gene; a red square node indicates a miRNA with upregulated expression in the recurrence group against the non-recurrence group, whereas a green square node indicates a downregulated miRNA in the recurrence group.

combination was first done in the GSE39040 dataset, and results showed that the optimal miRNA combination had the highest classification accuracy (89.2\%) when the number of miRNA was set at 15 . Further analysis of two-way hierarchical clustering was applied to samples in the GSE39040 database using the 15 miRNAs in the optimal combination, and the result was similar to that obtained using 78 significantly DEmiRs. Moreover, the SVM classifier constructed by these 15 miRNAs could accurately classify 58 of 65 samples in the GSE39040 database, and this sample classification effect was validated and evaluated in another two databases - GSE39052 and GSE79181 - which showed that the classifier could successfully categorize 22 samples in GSE39052 (84.62\%, 22/26) and 21 samples in GSE79181 $(91.3 \%, 21 / 23)$, indicating the sound performance of the SVM classifier constructed in the present study.

Kaplan-Meier survival analysis is one of the best options to measure the fraction of subjects living for a certain amount of time after treatment. ${ }^{42,43}$ In the present study, KaplanMeier survival analysis was first carried out according to the expression values of optimal feature miRNAs, the recurrence time of each corresponding sample, and the status (including recurrence and no recurrence) classified by the previously constructed SVM model, and the results showed that the survival time of the non-recurrence group was significantly longer than that of the recurrence group, and the decline rates of the survival ratio of the former group were significantly lower than that of the latter group. In addition, using expression values of the 15 optimal miRNAs as variables, their correlation with the recurrence time and sample status was analyzed by Cox regression, and four significantly correlated miRNAs - hsa-miR-10b, hsa-miR-1227, hsamiR-146b-3p, and hsa-miR-873 - were identified. The hsa-miR-10b is a particularly interesting candidate miRNA, given its close correlations with metastatic behaviors of breast cancer ${ }^{44}$ and gastric cancer. ${ }^{45}$ The hsa-miR-1227, a miRNA abundant in large oncosomes, can enhance migration of cancer-associated fibroblasts. ${ }^{46}$ A number of studies have examined the role of miR-146b microRNAs in cancer cell lines, one isoform of which, miR-146b-3p, was identified as a frequent constituent of six-miRNA SVM classifiers that could predict recurrence with a mean accuracy of $69 \%$; the expression level of miR-146b-3p in human non-small cell lung cancer was associated with the recurrence of this cancer postoperatively. ${ }^{47}$ The hsa-miR-873 is a novel tumor suppressor in ER-positive breast cancer ${ }^{48}$ and can inhibit tumorigenesis and metastasis of glioblastoma. ${ }^{49}$ This study suggests that these four miRNAs might be possible biomarkers for osteosarcoma recurrence.

It is well known that miRNAs are involved in cancer initiation and progression as well as in multiple metabolic pathways by regulating target gene expression. ${ }^{50,51}$ Due to the rapid development in bioinformatics and statistical genomics, gene network analysis has become a powerful approach that considers gene interactions and has been widely applied in gene expression studies of humans and 
A

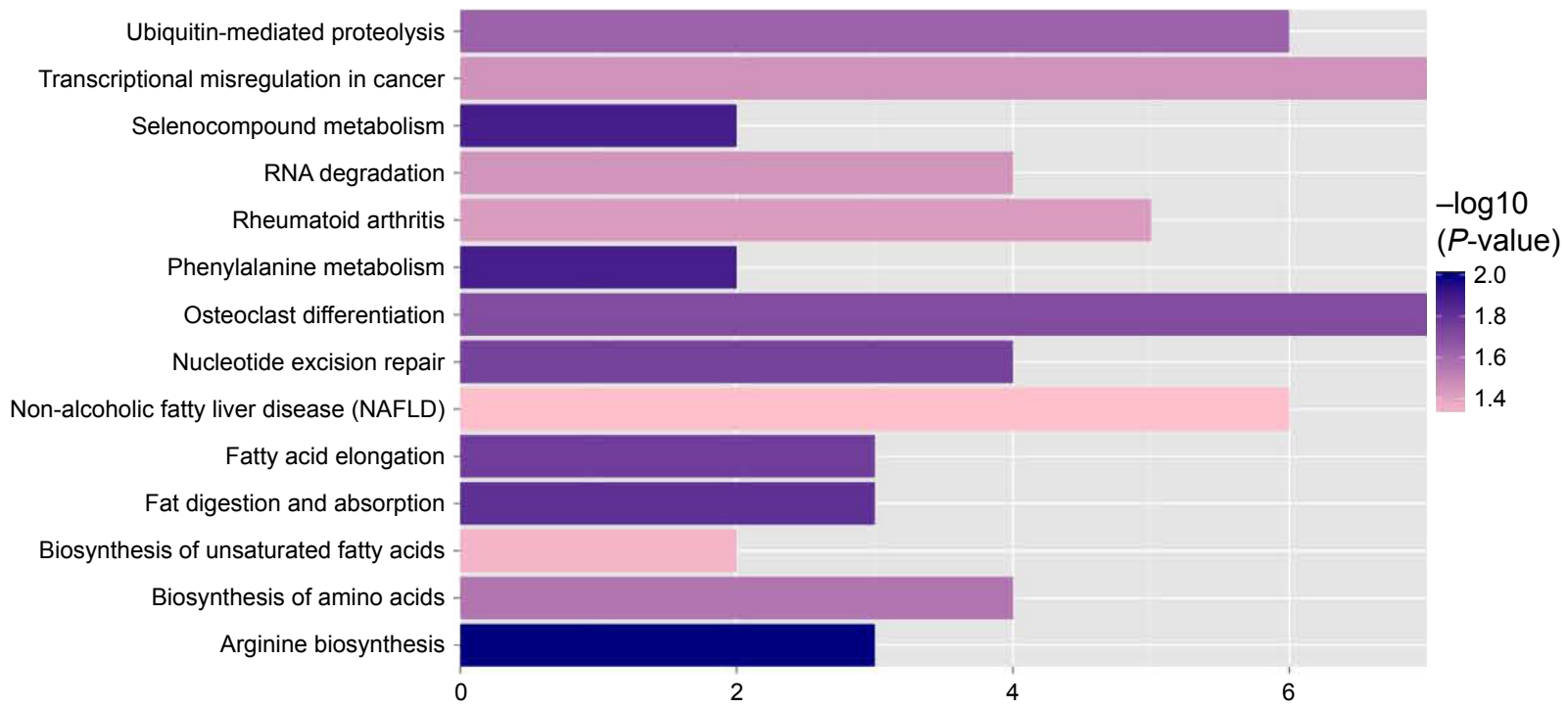

B

Transmembrane receptor protein serine/threonine kinase activity Sertoli cell development Serine/threonine protein kinase complex RNA polymerase II carboxy-terminal domain kinase activity Protein kinase complex

Positive regulation of stress-activated protein kinase signaling cascade Positive regulation of stress-activated MAPK cascade Paracrine signaling Negative regulation of protein complex assembly Lymphangiogenesis Lymph vessel morphogenesis Intrinsic apoptotic signaling pathway in response to hydrogen peroxide Intracellular receptor signaling pathway Feeding behavior Fatty acid elongation, saturated fatty acid

Fatty acid elongation Copper chaperone activity

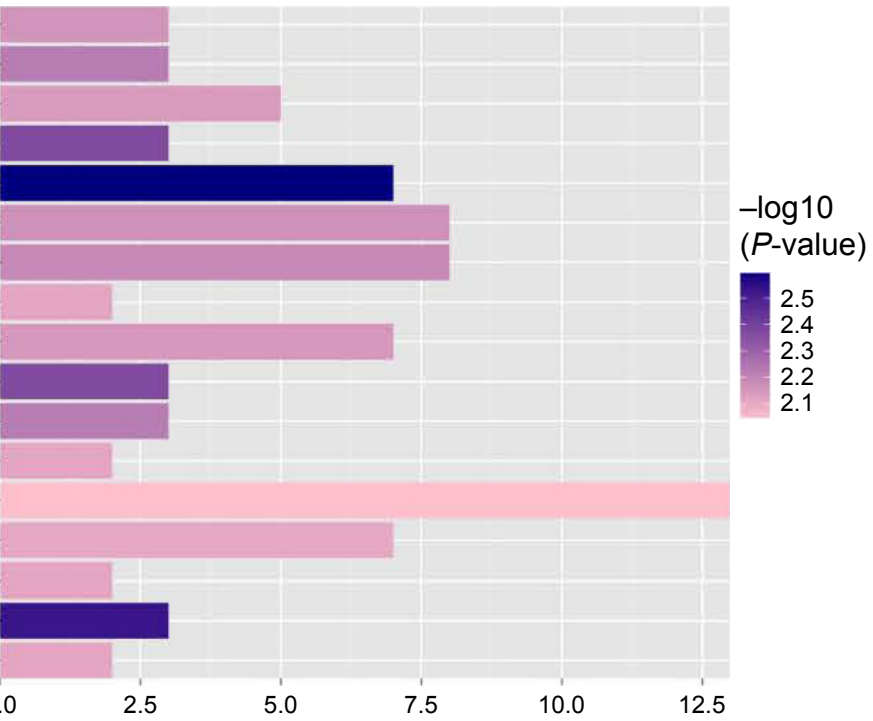

Figure 10 KEGG pathway (A) and column chart of GO function annotation (B) significantly related to target genes. Abbreviations: GO, gene ontology; KEGG, Kyoto Encyclopedia of Genes and Genomes.

model organisms. ${ }^{24,26}$ In the present study, 141 pairs of interaction links between hsa-miR-1227, hsa-miR-146b-3p, hsa-miR-873, and their target genes were obtained, and interaction networks were built by integrating the two kinds of connections, miRNA-gene and gene-gene. As genes that are highly interconnected within the network are usually involved in the same biological modules or pathways, functional annotations of the miRNA targeted genes according to $\mathrm{GO}$ and KEGG databases would provide ample numbers of candidate genes and more information about the mechanism of osteosarcoma. In total, $17 \mathrm{GO}$ functionally annotated terms and $14 \mathrm{KEGG}$ pathways were identified, of which one KEGG pathway was "Osteoclast differentiation", which was closely related to osteoblasts and osteosarcoma. ${ }^{52,53}$ There were a total of seven target genes involved in the "Osteoclast differentiation" pathway, including IL1A, CAMK4, TNFSF11, NCF4, PPP3CB, $M A P 3 K 7$, and SOCS3. The IL1A is known as a mediator of inflammation and a regulator of the blood-testis barrier, and participates in inhibiting Leydig cell steroidogenesis and stimulating Sertoli cell transfer. ${ }^{54}$ TNFSF11 is a member of the tumor necrosis factor family and plays essential roles in lymph node organogenesis, normal cellular immunity, osteoclastogenesis, and skeletal development. ${ }^{55}$ MAP3K7, also known as TGF $\beta$-associated kinase 1 (TAK1), is associated with early prostate-specific antigen recurrence in prostate cancer. ${ }^{56}$ SOCS3 is a cytokine-inducible inhibitor with critical, but selective, cell-specific effects, and also 


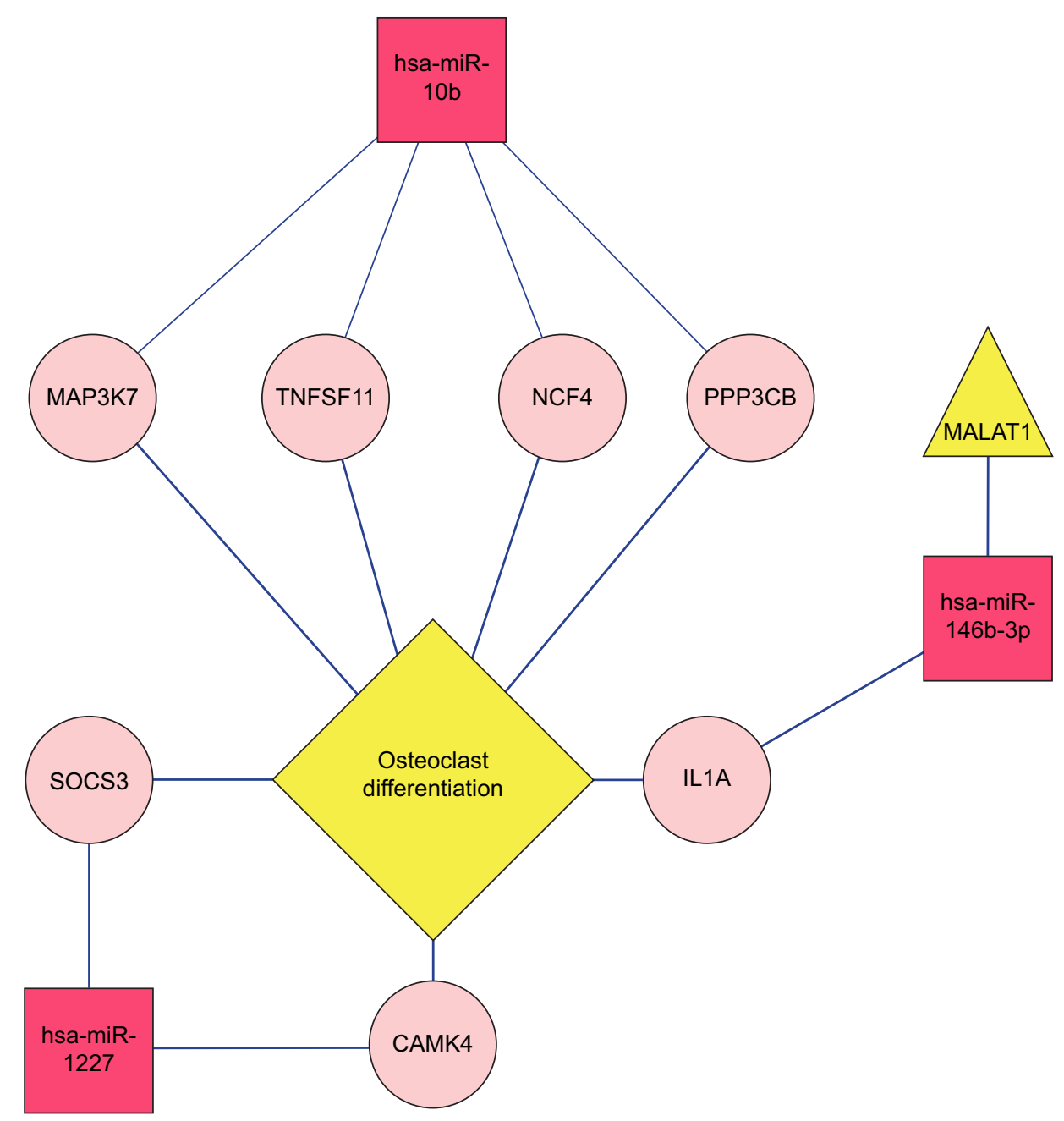

Figure II A regulatory network of IncRNA-miRNA-target gene pathway significantly related to osteosarcoma.

acts as an essential negative regulator of IL-23 signaling, the inhibition of which constrains the generation of Th17 differentiation. ${ }^{57}$ These target genes were validated to be targeted by hsa-miR-10b, hsa-miR-1227, and hsa-miR-146b-3p, separately. These results suggest that hsa-miR-10b, hsa-miR-1227, and hsa-miR-146b-3p might exert an effect on the osteoclast differentiation pathway via regulation of the seven target genes, thus affecting recurrence.

Considering lncRNAs may participate in the regulation of diverse cellular processes, such as cell proliferation and apoptosis, stem cell pluripotency, and development, ${ }^{10-12}$ a series of lncRNAs have been shown to be involved in the osteosarcoma. Emerging studies have suggested that several lncRNAs play a regulatory role in cellular processes via a complicated network involving mRNAs, miRNAs, and protein. ${ }^{58,59}$ Furthermore, networks involving lncRNAs, miRNAs, and mRNAs were explored in the present study. lncRNAs related to osteosarcoma were searched in the LncRNADisease database, ${ }^{31}$ which screened out three
lncRNAs - BC040587, ${ }^{34}$ LSAMP-AS3, ${ }^{35}$ and MALAT1. ${ }^{13}$ The regulatory relationship between osteosarcoma-related lncRNAs and miRNA-targeted genes was then built according to the previously obtained miRNAs relevant to this disease. The result showed that MALAT1 might directly regulate hsa-miR-146b-3p, which exerted a regulatory effect on the ILIA gene involved in the osteogenic differentiation pathway related to osteosarcoma. Previous studies have proved that MALAT1 could promote proliferation and metastasis of osteosarcoma cells by activating the PI3K_Akt pathway, ${ }^{13}$ and could be a potential therapeutic target in osteosarcoma. ${ }^{60}$ All of the above published studies prove the reliability of our present study and, therefore, the rest of the identified miRNAs and lncRNAs could be fresh factors related to the survival of patients with osteosarcoma. It should be mentioned that biological experiments were not carried out in this study. Further experimental studies using a large cohort of samples will be undertaken to validate the findings of this study. 


\section{Conclusion}

This study proposes a 15-miRNAs-based SVM classifier as a potential useful tool to predict osteosarcoma recurrence. The hsa-miR-10b, hsa-miR-1227, hsa-miR-146b-3p, and hsa-miR-873 miRNAs were closely associated with recurrence of osteosarcoma. Several pathways and GO functions significantly enriched with target genes of the four miRNAs were unraveled. LncRNA MALAT1 may directly regulate IL1A, involved in the osteoclast differentiation pathway, by targeting hsa-miR-146b-3p in osteosarcoma. Our results suggest possible mechanisms of osteosarcoma metastasis and recurrence, and provide DEmiRs which could be applied as potential biomarkers or therapeutic targets for osteosarcoma recurrences. More studies are warranted to verify the results of this study.

\section{Disclosure}

The authors report no conflicts of interest in this work.

\section{References}

1. Picci P. Osteosarcoma (osteogenic sarcoma). Orphanet J Rare Dis. 2007;2(6):1-4.

2. Weeden S, Grimer RJ, Cannon SR, Taminiau AH, Uscinska BM; European Osteosarcoma Intergroup. The effect of local recurrence on survival in resected osteosarcoma. Eur J Cancer. 2001;37(1):39-46.

3. Bartel DP. MicroRNAs: genomics, biogenesis, mechanism, and function. Cell. 2004;116(2):281-297.

4. Kobayashi E, Hornicek FJ, Duan Z. MicroRNA involvement in osteosarcoma. Sarcoma. 2012;2012:359739.

5. Yuan J, Lang J, Liu C, Zhou K, Chen L, Liu Y. The expression and function of miRNA-451 in osteosarcoma. Med Oncol. 2015;32(1):324.

6. Zhang H, Cai X, Wang Y, Tang H, Tong D, Ji F. microRNA-143, downregulated in osteosarcoma, promotes apoptosis and suppresses tumorigenicity by targeting Bcl-2. Oncol Rep. 2010;24(5):1363-1369.

7. Duan Z, Choy E, Harmon D, et al. MicroRNA-199a-3p is downregulated in human osteosarcoma and regulates cell proliferation and migration. Mol Cancer Ther. 2011;10(8):1337-1345.

8. Liu LH, Li H, Li JP, et al. miR-125b suppresses the proliferation and migration of osteosarcoma cells through down-regulation of STAT3. Biochem Biophys Res Commun. 2011;416(1-2):31-38.

9. Maire G, Martin JW, Yoshimoto M, Chilton-MacNeill S, Zielenska M, Squire JA. Analysis of miRNA-gene expression-genomic profiles reveals complex mechanisms of microRNA deregulation in osteosarcoma. Cancer Genet. 2011;204(3):138-146.

10. Deng Q, He B, Gao T, et al. Up-regulation of $91 \mathrm{H}$ promotes tumor metastasis and predicts poor prognosis for patients with colorectal cancer. PLoS One. 2014;9(7):e103022.

11. Gupta RA, Shah N, Wang KC, et al. Long non-coding RNA HOTAIR reprograms chromatin state to promote cancer metastasis. Nature. 2010;464(7291):1071-1076.

12. Prensner JR, Iyer MK, Sahu A, et al. The long noncoding RNA SChLAP1 promotes aggressive prostate cancer and antagonizes the SWI/SNF complex. Nat Genet. 2013;45(11):1392-1398.

13. Dong Y, Liang G, Yuan B, Yang C, Gao R, Zhou X. MALAT1 promotes the proliferation and metastasis of osteosarcoma cells by activating the PI3K/Akt pathway. Tumour Biol. 2015;36(3):1477-1486.

14. Zhang Q, Geng PL, Yin P, Wang XL, Jia JP, Yao J. Down-regulation of long non-coding RNA TUG1 inhibits osteosarcoma cell proliferation and promotes apoptosis. Asian Pac J Cancer Prev. 2013;14(4): 2311-2315.
15. Tian ZZ, Guo XJ, Zhao YM, Fang Y. Decreased expression of long non-coding RNA MEG3 acts as a potential predictor biomarker in progression and poor prognosis of osteosarcoma. Int J Clin Exp Pathol. 2015;8(11):15138-15142. eCollection 2015.

16. Zhou M, Wang X, Shi H, et al. Characterization of long non-coding RNA-associated ceRNA network to reveal potential prognostic lncRNA biomarkers in human ovarian cancer. Oncotarget. 2016;7(11): 12598-12611

17. Ma B, Li M, Zhang L, et al. Upregulation of long non-coding RNA TUG1 correlates with poor prognosis and disease status in osteosarcoma. Tumour Biol. 2016;37(4):4445-4455.

18. Zhao H, Hou W, Tao J, et al. Upregulation of lncRNA HNF1A-AS1 promotes cell proliferation and metastasis in osteosarcoma through activation of the Wnt/ $\beta$-catenin signaling pathway. Am J Transl Res. 2016;8(8):3503-3512. eCollection 2016.

19. Jendrzejewski J, He H, Radomska HS, et al. The polymorphism rs944289 predisposes to papillary thyroid carcinoma through a large intergenic noncoding RNA gene of tumor suppressor type. Proc Natl Acad Sci U S A. 2012;109(22):8646-8651.

20. Johnsson P, Ackley A, Vidarsdottir L, et al. A pseudogene long noncoding RNA network regulates PTEN transcription and translation in human cells. Nat Struct Mol Biol. 2013;20(4):440-446.

21. Liz J, Esteller M. IncRNAs and microRNAs with a role in cancer development. Biochim Biophys Acta. 2016;1859(1):169-176.

22. Tan JY, Sirey T, Honti F, et al. Extensive microRNA-mediated crosstalk between lncRNAs and mRNAs in mouse embryonic stem cells. Genome Res. 2015;25(5):655-666.

23. Cao MX, Jiang YP, Tang YL, Liang XH. The crosstalk between lncRNA and microRNA in cancer metastasis: orchestrating the epithelialmesenchymal plasticity. Oncotarget. 2017;8(7):12472-12483.

24. Lee I, Date SV, Adai AT, Marcotte EM. A probabilistic functional network of yeast genes. Science. 2004;306(5701):1555-1558.

25. Oldham MC, Konopka G, Iwamoto K, et al. Functional organization of the transcriptome in human brain. Nat Neurosci. 2008;11(11):1271-1282.

26. Ponomarev I, Wang S, Zhang L, Harris RA, Mayfield RD. Gene coexpression networks in human brain identify epigenetic modifications in alcohol dependence. J Neurosci. 2012;32(5):1884-1897.

27. Li J, Tseng GC. An adaptively weighted statistic for detecting differential gene expression when combining multiple transcriptomic studies. Ann Appl Stat. 2011;5(2A):994-1019.

28. Baur B, Bozdag S. A feature selection algorithm to compute gene centric methylation from probe level methylation data. PLoS One. 2016; 11(2):e0148977.

29. Hsu SD, Lin FM, Wu WY, et al. miRTarBase: a database curates experimentally validated microRNA-target interactions. Nucleic Acids Res. 2011;39(Database issue):D163-D169.

30. Chou CH, Chang NW, Shrestha S, et al. miRTarBase 2016: updates to the experimentally validated miRNA-target interactions database. Nucleic Acids Res. 2016;44(D1):D239-D247.

31. Chen G, Wang Z, Wang D, et al. LncRNADisease: a database for long-non-coding RNA-associated diseases. Nucleic Acids Res. 2013; 41(Database issue):D983-D986.

32. Robinson MD, McCarthy DJ, Smyth GK. edgeR: a Bioconductor package for differential expression analysis of digital gene expression data. Bioinformatics. 2010;26(1):139-140.

33. Ge Y, Dudoit S, Speed TP. Resampling-based multiple testing for microarray data analysis. Test. 2003;12(1):1-77.

34. Pasic I, Shlien A, Durbin AD, et al. Recurrent focal copy-number changes and loss of heterozygosity implicate two noncoding RNAs and one tumor suppressor gene at chromosome 3 q13.31 in osteosarcoma. Cancer Res. 2010;70(1):160-171.

35. Lipovich L, Johnson R, Lin CY. MacroRNA underdogs in a microRNA world: evolutionary, regulatory, and biomedical significance of mammalian long non-protein-coding RNA. Biochim Biophys Acta. 2010; 1799(9):597-615.

36. Wang Z, Wang J, Yang Y, et al. Loss of hsa-miR-337-3p expression is associated with lymph node metastasis of human gastric cancer. $J$ Exp Clin Cancer Res. 2013;32:76. 
37. Wang M, Li C, Nie H, et al. Down-regulated miR-625 suppresses invasion and metastasis of gastric cancer by targeting ILK. FEBS Lett. 2012;586(16):2382-2388

38. Li Z, Cao Y, Jie Z, et al. miR-495 and miR-551a inhibit the migration and invasion of human gastric cancer cells by directly interacting with PRL-3. Cancer Lett. 2012;323(1):41-47.

39. Brown MP, Grundy WN, Lin D, et al. Knowledge-based analysis of microarray gene expression data by using support vector machines. Proc Natl Acad Sci US A. 2000;97(1):262-267.

40. Furey TS, Cristianini N, Duffy N, Bednarski DW, Schummer M, Haussler D. Support vector machine classification and validation of cancer tissue samples using microarray expression data. Bioinformatics. 2000;16(10):906-914.

41. Mukherjee S, Rifkin R. Support Vector Machine Classification of Microarray Data. AI Memo 1677. Cambridge, MA: Massachusetts Institute of Technology. 2010;1(5):1-4.

42. Goel MK, Khanna P, Kishore J. Understanding survival analysis Kaplan-Meier estimate. Int J Ayurveda Res. 2010;1(4):274-278.

43. May WL. Kaplan-Meier survival analysis. In: Schwab M, editor. Encyclopedia of Cancer. Berlin Heidelberg: Springer; 2008.

44. Ma L. Role of miR-10b in breast cancer metastasis. Breast Cancer Res. 2010;12(5):210.

45. Li Z, Lei H, Luo M, et al. DNA methylation downregulated mir-10b acts as a tumor suppressor in gastric cancer. Gastric Cancer. 2015; 18(1):43-54.

46. Morello M, Minciacchi VR, de Candia P, et al. Large oncosomes mediate intercellular transfer of functional microRNA. Cell Cycle. 2013;12(22):3526-3536

47. Patnaik SK, Kannisto E, Mallick R, Yendamuri S. Overexpression of the lung cancer-prognostic miR-146b microRNAs has a minimal and negative effect on the malignant phenotype of A549 lung cancer cells. PLoS One. 2011;6(7):e22379.

48. Cui J, Yang Y, Li H, et al. MiR-873 regulates ER $\alpha$ transcriptional activity and tamoxifen resistance via targeting CDK3 in breast cancer cells. Oncogene. 2015;34(30):3895-3907.
49. Wang RJ, Li JW, Bao BH, et al. MicroRNA-873 (miRNA-873) inhibits glioblastoma tumorigenesis and metastasis by suppressing the expression of IGF2BP1. J Biol Chem. 2015;290(14):8938-8948.

50. Calin GA, Croce CM. MicroRNA-cancer connection: the beginning of a new tale. Cancer Res. 2006;66(15):7390-7394.

51. Paul P, Chakraborty A, Sarkar D, et al. Interplay between miRNAs and human diseases: a review. J Cell Physiol. Epub 2017 Feb 9.

52. Boyle WJ, Simonet WS, Lacey DL. Osteoclast differentiation and activation. Nature. 2003;423(6937):337-342.

53. Thomas DM, Johnson SA, Sims NA, et al. Terminal osteoblast differentiation, mediated by runx 2 and $\mathrm{p} 27 \mathrm{KIP} 1$, is disrupted in osteosarcoma. J Cell Biol. 2004;167(5):925-934.

54. Sarkar O, Mathur PP, Cheng CY, Mruk DD. Interleukin 1 alpha (IL1A) is a novel regulator of the blood-testis barrier in the rat. Biol Reprod. 2008;78(3):445-454.

55. Odgren PR, Kim N, Mackay CA, Mason-Savas A, Choi Y, Marks SC Jr. The role of RANKL (TRANCE/TNFSF11), a tumor necrosis factor family member, in skeletal development: effects of gene knockout and transgenic rescue. Connect Tissue Res. 2003;44(Suppl 1):264-271.

56. Kluth M, Hesse J, Heinl A, et al. Genomic deletion of MAP3K7 at 6q12-22 is associated with early PSA recurrence in prostate cancer and absence of TMPRSS2:ERG fusions. Mod Pathol. 2013;26(7):975-983.

57. Chen Z, Laurence A, Kanno Y, et al. Selective regulatory function of Socs 3 in the formation of IL-17-secreting T cells. Proc Natl Acad Sci US A. 2006;103(21):8137-8142.

58. Ernst C, Morton CC. Identification and function of long non-coding RNA. Front Cell Neurosci. 2013;7:168.

59. Yang G, Lu X, Yuan L. LncRNA: a link between RNA and cancer. Biochim Biophys Acta. 2014;1839(11):1097-1109.

60. Cai X, Liu Y, Yang W, et al. Long noncoding RNA MALAT1 as a potential therapeutic target in osteosarcoma. J Orthop Res. 2016;34(6): 932-941. 


\section{Supplementary materials}

Table SI Screened 78 miRNAs with significant differential expression

\begin{tabular}{lllll}
\hline miRNA_ID & $\mathbf{P}$-value & $\mathbf{Q}$ & $\mathbf{Q p}$ & tau $^{2}$ \\
\hline hsa-miR-432 & 0.00002 & 0.6704 & 0.7152 & 0 \\
hsa-miR-329 & 0.00002 & 0.2139 & 0.8986 & 0 \\
hsa-miR-889 & 0.0002 & 0.7548 & 0.6857 & 0 \\
hsa-miR-337-3p & 0.0002 & 0.0778 & 0.9619 & 0 \\
hsa-miR-635 & 0.0004 & 0.4406 & 0.8023 & 0 \\
hsa-miR-296-3p & 0.0004 & 1.4533 & 0.4835 & 0 \\
hsa-miR-548c-3p & 0.0006 & 0.8417 & 0.6565 & 0 \\
hsa-miR-625 & 0.0006 & 0.0724 & 0.9644 & 0 \\
hsa-miR-55Ia & 0.0006 & 0.2312 & 0.8908 & 0 \\
hsa-miR-5I0 & 0.0006 & 0.0666 & 0.9672 & 0 \\
hsa-miR-I0b & 0.0009 & 1.6776 & 0.4322 & 0 \\
hsa-miR-375 & 0.0015 & 1.2804 & 0.5272 & 0 \\
hsa-miR-586 & 0.0017 & 0.1737 & 0.9168 & 0 \\
hsa-miR-2II & 0.0018 & 1.2030 & 0.5480 & 0 \\
hsa-miR-338-3p & 0.0030 & 0.3902 & 0.8227 & 0 \\
hsa-miR-509-3-5p & 0.0030 & 0.5238 & 0.7696 & 0 \\
hsa-miR-60I & 0.0032 & 0.8572 & 0.6514 & 0 \\
hsa-miR-506 & 0.0035 & 0.1168 & 0.9433 & 0 \\
hsa-miR-493 & 0.0036 & 0.8930 & 0.6399 & 0 \\
hsa-miR-382 & 0.0052 & 0.4549 & 0.7966 & 0 \\
hsa-miR-448 & 0.0052 & 0.3315 & 0.8473 & 0 \\
hsa-miR-485-3p & 0.0056 & 1.9759 & 0.3723 & 0 \\
hsa-miR-330-3p & 0.0061 & 1.7235 & 0.4224 & 0 \\
hsa-miR-885-5p & 0.0062 & 0.4348 & 0.8046 & 0 \\
hsa-miR-154 & 0.0067 & 0.5234 & 0.7697 & 0 \\
hsa-miR-323-3p & 0.0073 & 1.4660 & 0.4805 & 0 \\
hsa-miR-38I & 0.0081 & 1.0856 & 0.5811 & 0 \\
hsa-miR-I46b-3p & 0.0083 & 0.1552 & 0.9253 & 0 \\
hsa-miR-I324 & 0.0092 & 0.2516 & 0.8818 & 0 \\
hsa-miR-544 & 0.0095 & 0.2217 & 0.8951 & 0 \\
hsa-miR-342-3p & 0.0099 & 1.7962 & 0.4073 & 0 \\
hsa-miR-944 & 0.0122 & 0.2137 & 0.8987 & 0 \\
hsa-miR-409-3p & 0.0124 & 0.3380 & 0.8445 & 0 \\
hsa-miR-362-3p & 0.0128 & 1.5226 & 0.4671 & 0 \\
hsa-miR-520a-3p & 0.0129 & 1.0973 & 0.5777 & 0 \\
hsa-miR-299-5p & 0.0141 & 0.9906 & 0.6094 & 0 \\
hsa-miR-99b & 0.0145 & 1.2373 & 0.5387 & 0 \\
hsa-miR-576-3p & 0.0198 & 1.0569 & 0.5895 & 0 \\
\hline & & & & $(C 0 n t i n u e d)$
\end{tabular}

Table SI (Continued)

\begin{tabular}{|c|c|c|c|c|}
\hline miRNA_ID & $P$-value & $\mathbf{Q}$ & Qp & $\operatorname{tau}^{2}$ \\
\hline hsa-miR-636 & 0.0202 & 0.9612 & 0.6184 & 0 \\
\hline hsa-miR-3I & 0.0207 & 1.4158 & 0.4927 & 0 \\
\hline hsa-miR-543 & 0.0219 & 0.0557 & 0.9725 & 0 \\
\hline hsa-miR-509-5p & 0.0233 & 0.2104 & 0.9002 & 0 \\
\hline hsa-miR-873 & 0.0237 & 0.1581 & 0.9240 & 0 \\
\hline hsa-miR-572 & 0.0245 & 0.2224 & 0.8947 & 0 \\
\hline hsa-miR-I48b & $0.025 I$ & 1.7011 & 0.4272 & 0 \\
\hline hsa-miR-324-5p & 0.0262 & 1.6948 & 0.4285 & 0 \\
\hline hsa-miR-422a & 0.0262 & 0.3929 & 0.8217 & 0 \\
\hline hsa-miR-I8Ia & 0.0269 & 0.5740 & 0.7505 & 0 \\
\hline hsa-miR-25 & 0.0276 & 1.7530 & 0.4162 & 0 \\
\hline hsa-miR-29c & 0.0286 & 0.3219 & 0.8513 & 0 \\
\hline hsa-miR-5I7c & 0.0290 & 0.7942 & 0.6723 & 0 \\
\hline hsa-miR-50I-5p & 0.0296 & 0.7887 & $0.674 I$ & 0 \\
\hline hsa-miR-639 & 0.0296 & $0.644 I$ & 0.7247 & 0 \\
\hline hsa-miR-I 275 & 0.0296 & 0.2700 & 0.8737 & 0 \\
\hline hsa-miR-129-3p & 0.0326 & 0.6742 & 0.7138 & 0 \\
\hline hsa-miR-92I & 0.0327 & 0.3280 & 0.8487 & 0 \\
\hline hsa-miR-I24 & 0.0338 & 0.7222 & 0.6969 & 0 \\
\hline hsa-miR-I47 & 0.0358 & 0.4122 & 0.8138 & 0 \\
\hline hsa-miR-28-5p & 0.0362 & 0.4770 & 0.7878 & 0 \\
\hline hsa-miR-200c & 0.0365 & 0.9670 & 0.6166 & 0 \\
\hline hsa-miR-5I8f & 0.0382 & 1.0540 & 0.5904 & 0 \\
\hline hsa-miR-I34 & 0.0390 & 1.2245 & 0.5421 & 0 \\
\hline hsa-miR-29a & 0.0395 & 0.4061 & 0.8162 & 0 \\
\hline hsa-miR-337-5p & 0.0399 & 0.9856 & 0.6109 & 0 \\
\hline hsa-miR-50I-3p & 0.0402 & 1.0533 & 0.5906 & 0 \\
\hline hsa-miR-139-3p & 0.0407 & 1.7569 & 0.4154 & 0 \\
\hline hsa-miR-I22 & 0.0425 & 0.0150 & 0.9925 & 0 \\
\hline hsa-miR-43I & 0.0425 & 0.0928 & 0.9546 & 0 \\
\hline hsa-miR-630 & 0.0436 & $0.817 \mid$ & 0.6646 & 0 \\
\hline hsa-miR-I 227 & 0.0437 & 0.4731 & 0.7894 & 0 \\
\hline hsa-miR-I36 & $0.044 I$ & 0.4049 & 0.8167 & 0 \\
\hline hsa-miR-127-3p & 0.0470 & 0.2269 & 0.8928 & 0 \\
\hline hsa-miR-204 & 0.0471 & 0.1403 & 0.9322 & 0 \\
\hline hsa-miR-36I-3p & $0.047 I$ & 0.5252 & 0.7691 & 0 \\
\hline hsa-miR-720 & 0.0488 & 0.1208 & 0.9414 & 0 \\
\hline hsa-miR-342-5p & 0.0490 & 0.5989 & 0.7412 & 0 \\
\hline hsa-miR-885-3p & 0.0494 & 0.8097 & $0.667 I$ & 0 \\
\hline hsa-miR-302d & 0.0497 & 0.5082 & 0.7756 & 0 \\
\hline
\end{tabular}


Table S2 GO annotation significantly related to target genes

\begin{tabular}{|c|c|c|c|}
\hline Term & Count & P-value & Genes \\
\hline Lymphangiogenesis & 3 & 0.0043 & PPP3CB, FLT4, BMPR2 \\
\hline Transmembrane receptor protein serine/threonine kinase activity & 3 & 0.0069 & LTBP4, LTBPI, BMPR2 \\
\hline Feeding behavior & 7 & 0.0078 & MCHRI, PPYRI, OPRDI, GLS, ASIP, PHF2IA, IAPP \\
\hline RNA polymerase II carboxy-terminal domain kinase activity & 3 & 0.0043 & GTF2HI, CDK9, GTF2H4 \\
\hline Copper chaperone activity & 2 & 0.0077 & PARK7, CCS \\
\hline Fatty acid elongation, saturated fatty acid & 2 & 0.0077 & ELOVL4, ELOVL6 \\
\hline Fatty acid elongation & 3 & 0.0029 & ELOVL2, ELOVL4, ELOVL6 \\
\hline Intracellular receptor signaling pathway & 13 & 0.0090 & $\begin{array}{l}\text { ZNF536, CCNEI, DCBLD2, PGR, MAP3K7, GPR56, ITCH, } \\
\text { NODI, KANK2, THRA, PARK7, RXRB, DDX5 }\end{array}$ \\
\hline Negative regulation of protein complex assembly & 7 & 0.0071 & THRA, HEY2, SPTANI, MAP3K7, OPRD I, MAPREI, PARK7 \\
\hline Positive regulation of stress-activated MAPK cascade & 8 & 0.0064 & $\begin{array}{l}\text { ILIA, FLT4, MAP3KIO, TNFSFII, NODI, HIPK2, MAP3K7, } \\
\text { STK } 25\end{array}$ \\
\hline Lymph vessel morphogenesis & 3 & 0.0059 & PPP3CB, FLT4, BMPR2 \\
\hline Intrinsic apoptotic signaling pathway in response to hydrogen peroxide & 2 & 0.0077 & PARK7, STK25 \\
\hline Paracrine signaling & 2 & 0.0077 & TNFSFII, PGR \\
\hline Sertoli cell development & 3 & 0.0059 & DMRTI, ARID4B, SDCI \\
\hline Positive regulation of stress-activated protein kinase signaling cascade & 8 & 0.0067 & $\begin{array}{l}\text { ILIA, FLT4, MAP3KIO, TNFSFII, NODI, HIPK2, MAP3K7, } \\
\text { STK } 25\end{array}$ \\
\hline Serine/threonine protein kinase complex & 5 & 0.0072 & GTF2HI, CDK9, MAP3K7, RBICCI, GTF2H4 \\
\hline Protein kinase complex & 7 & 0.0026 & $\begin{array}{l}\text { GTF2HI, CDK9, CCNEI, PRKAB2, GTF2H4, MAP3K7, } \\
\text { RBICCI }\end{array}$ \\
\hline
\end{tabular}

Abbreviation: GO, gene ontology.

Table S3 The KEGG pathway significantly related to target genes

\begin{tabular}{llll}
\hline Term & Count & P-value & Genes \\
\hline Arginine biosynthesis & 3 & 0.0100 & ARG2, GOT2, GLS \\
Fatty acid elongation & 3 & 0.017 I & ELOVL2, ELOVL4, ELOVL6 \\
Nucleotide excision repair & 4 & 0.0178 & GTF2HI, LIGI, RAD23B, GTF2H4 \\
Osteoclast differentiation & 7 & 0.0195 & ILIA, CAMK4, TNFSFII, NCF4, PPP3CB, MAP3K7, SOCS3 \\
Rheumatoid arthritis & 5 & 0.0379 & ITGAL, TNFSFII, IL8, MMP3, ILIA \\
Phenylalanine metabolism & 2 & 0.0128 & GLYAT, GOT2 \\
Selenocompound metabolism & 2 & SEPHSI, TXNRDI \\
Fat digestion and absorption & 3 & 0.0128 & PNLIPRPI, ABCAI, GOT2 \\
Ubiquitin-mediated proteolysis & 6 & 0.0152 & PIAS3, CBL, TRIM32, ITCH, TRIM37, SOCS3 \\
Biosynthesis of amino acids & 4 & 0.0235 & ARG2, GOT2, RPE, MAT2B \\
Transcriptional misregulation in cancer & 7 & 0.0276 & CDK9, MMP3, IL8, PAX5, RXRB, CCNAI, DDX5 \\
RNA degradation & 4 & $0.035 I$ & DDX6, CNOT6, I0200, CNOT4 \\
Biosynthesis of unsaturated fatty acids & 2 & 0.0354 & ELOVL2, ELOVL6 \\
Non-alcoholic fatty liver disease (NAFLD) & 6 & 0.0459 & ILIA, PRKAB2, IL8, ITCH, NDUFA4L2, SOCS3
\end{tabular}

Abbreviation: KEGG, Kyoto Encyclopedia of Genes and Genomes.

OncoTargets and Therapy

\section{Publish your work in this journal}

OncoTargets and Therapy is an international, peer-reviewed, open access journal focusing on the pathological basis of all cancers, potential targets for therapy and treatment protocols employed to improve the management of cancer patients. The journal also focuses on the impact of management programs and new therapeutic agents and protocols on
Dovepress

patient perspectives such as quality of life, adherence and satisfaction. The manuscript management system is completely online and includes a very quick and fair peer-review system, which is all easy to use. Visit http://www.dovepress.com/testimonials.php to read real quotes from published authors. 